\title{
Nosetip Bluntness Effects on Transition at Hypersonic Speeds: Experimental and Numerical Analysis Under NATO STO AVT-240
}

\author{
Pedro Paredes, ${ }^{*}$ Meelan M. Choudhari, ${ }^{\dagger}$ Fei Li, ${ }^{\ddagger}$ \\ NASA Langley Research Center, Hampton, VA 23681, USA \\ Joseph S. Jewell,,$^{\S}$ Roger L. Kimmel, \\ U.S. Air Force Research Laboratory, Wright-Patterson Air Force Base, OH 45433, USA \\ Eric C. Marineau, \\ AEDC White Oak, Silver Spring, MD 20903, USA \\ Guillaume Grossir** \\ von Karman Institute for Fluid Dynamics, Chaussée de Waterloo 72, 1640 Rhode-St-Genèse, Belgium
}

\begin{abstract}
The existing database of transition measurements in hypersonic ground facilities has established that the onset of boundary layer transition over a circular cone at zero angle of attack shifts downstream as the nosetip bluntness is increased with respect to a sharp cone. However, this trend is reversed at sufficiently large values of the nosetip Reynolds number, so that the transition onset location eventually moves upstream with a further increase in nosetip bluntness. This transition reversal phenomenon, which cannot be explained on the basis of linear stability theory, was the focus of a collaborative investigation under the NATO STO group AVT-240 on Hypersonic Boundary-Layer Transition Prediction. The current paper provides an overview of that effort, which included wind tunnel measurements in three different facilities and theoretical analysis related to modal and nonmodal amplification of boundary layer disturbances. Because neither first and secondmode waves nor entropy-layer instabilities are found to be substantially amplified to initiate transition at large bluntness values, transient (i.e., nonmodal) disturbance growth has been investigated as the potential basis for a physics-based model for the transition reversal phenomenon. Results of the transient growth analysis indicate that disturbances that are initiated within the nosetip or in the vicinity of the juncture between the nosetip and the frustum can undergo relatively significant nonmodal amplification and that the maximum energy gain increases nonlinearly with the nose radius of the cone. This finding does not provide a definitive link between transient growth and the onset of transition, but it is qualitatively consistent with the experimental observations that frustum transition during the reversal regime was highly sensitive to wall roughness, and furthermore, was dominated by disturbances that originated near the nosetip.
\end{abstract}

\footnotetext{
*NASA NPP Fellow, Computational AeroSciences Branch. AIAA Member

${ }^{\dagger}$ Research Scientist, Computational AeroSciences Branch. AIAA Associate Fellow

${ }^{\ddagger}$ Research Scientist, Computational AeroSciences Branch.

$\S$ Research Scientist (Spectral Energies, LLC), AFRL/RQHF. Senior AIAA Member

๑ Principal Aerospace Engineer, AFRL/RQHF. AIAA Associate Fellow

$\|_{\text {Chief Technologist. Senior AIAA Member }}$

** Post-doctoral Fellow, Aeronautics and Aerospace Department
} 


\section{Nomenclature}

\begin{tabular}{|c|c|c|c|}
\hline E & total energy norm & $\kappa$ & streamwise curvature \\
\hline K & kinetic energy norm & $\rho$ & density \\
\hline$G^{\text {mean }}$ & mean energy gain & $(\xi, \eta, \zeta)$ & streamwise, wall-normal and \\
\hline$G^{\text {out }}$ & outlet energy gain & & spanwise coordinates \\
\hline$h_{1}$ & streamwise metric factor & $\Delta \xi$ & optimal growth interval \\
\hline$h_{3}$ & spanwise metric factor & $\phi$ & angular coordinate \\
\hline$h_{t}$ & total enthalpy & $\theta$ & cone half-angle \\
\hline$J$ & objective function & $\mathbf{M}$ & energy weight matrix \\
\hline$k$ & peak-to-valley roughness height & Subscript & \\
\hline$L$ & reference length & $\infty$ & freestream value \\
\hline$m$ & azimuthal wavenumber & 0 & $\begin{array}{l}\text { freestream value } \\
\text { initial position }\end{array}$ \\
\hline$M$ & Mach number & 1 & initial position \\
\hline$N$ & Logarithmic amplification factor & $\begin{array}{l}1 \\
i\end{array}$ & final position \\
\hline$\overline{\mathbf{q}}$ & vector of base flow variables & $j$ & \\
\hline$\tilde{\mathbf{q}}$ & vector of perturbation variables & $T$ & transition location \\
\hline$\hat{\mathbf{q}}$ & vector of amplitude variables & Superscripts & \\
\hline$R e_{k k}$ & roughness-height Reynolds number & $*$ & dimensional value \\
\hline$R e_{\infty}$ & freestream unit Reynolds number & $H$ & conjugate transpose \\
\hline $\operatorname{Re}_{R_{N}}$ & nosetip Reynolds number & Abbreviations & \\
\hline $\begin{array}{l}R_{\xi_{T}} \\
r_{b}\end{array}$ & $\begin{array}{l}\text { transition Reynolds number } \\
\text { local radius }\end{array}$ & AFRL & Air Force Research Laboratory \\
\hline$R_{N}$ & nose radius & AEDC & Arnold Engineering Development Comple \\
\hline$T$ & temperature & DNS & direct numerical simulation \\
\hline$T_{\text {wall }}$ & wall temperature & DPLR & data parallel-line relaxation \\
\hline$T_{\text {wall }, \text { ad }}$ & adiabatic wall temperature & FST & freestream turbulence \\
\hline$(u, v, w)$ & streamwise, wall-normal and & NS & Navier-Stokes \\
\hline & spanwise velocity components & LIF & laser-induced fluorescence \\
\hline$(x, y, z)$ & Cartesian coordinates & PSE & parabolized stability equations \\
\hline
\end{tabular}

\section{Introduction}

Laminar-turbulent transition of boundary-layer flows can have a strong impact on the performance of hypersonic vehicles because of its influence on the surface skin friction and aerodynamic heating. Therefore, the prediction and control of transition onset and the associated variation in aerothermodynamic parameters in high-speed flows is a key issue for optimizing the performance of the next-generation aerospace vehicles. Although many practical aerospace vehicles are blunt, the mechanisms that lead to boundary-layer instability and transition on blunt geometries are not well understood as yet. A detailed review of boundary layer transition over sharp and blunt cones in a hypersonic freestream is given by Schneider [1]. As described therein, both experimental and numerical studies have shown that the modal growth of Mack-mode instabilities (or, equivalently, the so called second-mode waves) is responsible for laminar-turbulent transition on sharp, axisymmetric cones at zero angle of attack. Studies have also shown that increased nosetip bluntness has a stabilizing effect on the amplification of Mack-mode instabilities, which is consistent with the observation that the onset of transition is displaced downstream as the nose bluntness is increased. However, while the boundary layer flow continues to become more stable with increasing nose bluntness, experiments indicate that the downstream movement in transition actually slows down and eventually reverses as the nose bluntness exceeds a certain critical range of values. The observed reversal in transition onset at large values of nose bluntness is contrary to the predictions of linear stability theory, and therefore, must be explained using a different paradigm. While no satisfactory explanation has been proposed as yet, one of the physical effects that have been suspected to cause this transition reversal is the role of surface roughness.

Earlier measurements related to the effect of nose bluntness on frustum transition over hypersonic blunt cones have been thoroughly documented by Stetson. ${ }^{2}$ He concluded that the details of the nose tip flow played an important role in the transition reversal process, even though the onset of transition occurred 
significantly farther downstream over the frustum of the cone. Stetson ${ }^{2}$ also observed that the measured transition locations within this regime were not easily reproducible across different runs. At a fixed set of freestream conditions, transition onset was found to vary across a wide range of frustum stations, and at times, the boundary layer flow remained laminar over the entire cone. Nonaxisymmetric transition patterns were observed even at zero angle of attack, and the measured length of the transition zone was much larger than that for cones with smaller values of nose bluntness. Finally, Stetson observed that frustum transition in the transition-reversal regime was highly sensitive to surface roughness in the nosetip region. For smaller nosetip bluntness prior to transition reversal, the surface finish on the nosetip (or the frustum) appeared to have no effect on frustum transition. Polishing the blunt nosetip before the wind tunnel run for the large bluntness cones resulted in either higher frustum transition Reynolds numbers or a completely laminar flow over the model. Primarily on the basis of this last observation, Stetson speculated that frustum transition for large bluntness cones was dominated by disturbances originating near the nosetip. Therefore, roughnessinduced transient growth appears to be a reasonable explanation for the transition-reversal phenomena in large bluntness cones at high speeds.

Historically, the term "bypass transition" has been used to identify transition paths that cannot be explained via modal amplification of small-amplitude disturbances. ${ }^{3}$ Well-known examples of bypass transition include the transition due to high levels of freestream disturbances, as for example, in turbomachinery, or the subcritical transition observed in Poiseuille pipe flow experiments, ${ }^{4-6}$ transition due to distributed surface roughness on flat plates ${ }^{7,8}$ or cones, ${ }^{9}$ and subcritical transition observed on spherical forebodies. ${ }^{10-13}$ Because of the strongly favorable pressure gradient over blunt bodies such as hemispherical nose tips and spherical segment capsules, the laminar boundary layer is highly stable; and hence, the observed onset of transition on such bodies has been known as the "blunt-body paradox". In recent years, the phenomenon of transient, nonmodal amplification of disturbance energy has emerged as a possible explanation for many cases of bypass transition. Mathematically, the transient, nonmodal growth is associated with the nonorthogonality of the eigenvectors corresponding to the linear disturbance equations. Physically, the main growth mechanism corresponds to the lift-up effect, ${ }^{14-16}$ which results from the conservation of horizontal momentum in the course of spanwise varying wall-normal displacement of the fluid particles. The actual growth in any given scenario is determined by the details of the external disturbance environment. However, an upper bound on the magnitude of energy gain via transient growth can be predicted by using the so-called optimal growth theory, which is typically formulated to maximize the disturbance growth across a specified interval of streamwise locations. Regardless of the flow Mach number, ${ }^{17,18}$ the disturbances experiencing the highest magnitude of transient growth have been found to be stationary streaks that arise from initial perturbations in the form of streamwise vortices. Schmid \& Henningson ${ }^{19}$ and $\mathrm{Schmid}^{20}$ provide a thorough review of the transient growth theory and the earlier results from the literature.

Reshotko \& Tumin ${ }^{21}$ were able to successfully correlate the transition data from several wind tunnel experiments involving subcritical, nose tip transition by using a semiempirical correlation derived from the optimal transient growth theory, which provides an upper bound on the magnitude of transient growth under suitable constraints. Reshotko \& Tumin ${ }^{21}$ extended the ideas from Anderson et al., ${ }^{22}$ who had investigated subcritical transition in a flat plate boundary layer due to moderate to high levels of freestream turbulence (FST) and had derived a correlation based on the optimal growth of boundary layer disturbances generated by the FST. Based on the hypothesis that a similar disturbance growth could also be initiated by distributed surface roughness over hypersonic blunt forebodies, Reshotko \& Tumin ${ }^{21}$ were able to develop a semiempirical transition correlation by linking a critical disturbance amplitude required for the onset of transition with the roughness height parameter via a gain function based on the optimal growth framework. Even though the relevance of the linear, optimal disturbance growth concept to realistic, rough nose tips remains to be established, their work provides the first physics-based model toward a potential resolution of the bluntbody paradox. Unlike other established models based on empirical curve fits that are valid for a specific subclass of datasets, Reshotko \& Tumin's optimal-growth-based transition criterion has been able to provide a reasonable correlation with the measurements in various wind tunnel and ballistic range facilities and for a broad range of surface temperature ratios. In follow-on work, Paredes et al. ${ }^{23,24}$ have revised the Reshotko \& Tumin correlation by including the effects of nonparallel basic state evolution, curvature terms, and the variation of both inflow and outflow locations for the transient growth interval. Their results indicate that application of a more thorough theoretical framework reveals certain new features of optimal growth characteristics that were not indicated by the parallel framework used in the previous correlation. However, despite these changes, the constants in the transition correlation remain close to their original values. 
Not withstanding the questions related to the physical relevance of optimal growth theory, the successful correlation of much of the available data by the Reshotko \& Tumin correlation raises the possibility that a similar framework could also correlate (or, perhaps, explain) the observations of transition reversal over blunt cones. That possibility was investigated during a collaborative efforts under the NATO STO group AVT240 on Hypersonic Boundary-Layer Transition Prediction focused on the problem of blunt cone transition and the potential role of transient growth in the transition reversal phenomenon. While the problems of blunt-body paradox and transition reversal over blunt cones share a key similarity by way of transition onset in the absence of (significant) modal instability, they also exhibit two major differences. First, the onset of transition in the latter case is typically observed over the frustum of the cone, as opposed to the nose tip in the case of blunt body paradox. As such, the transient growth characteristics of blunt cone boundary layers are also expected to be different, and in fact, more complex than those over a blunt nose without any frustum. Besides investigating the transient growth features over blunt cones, the NATO group's effort also included wind tunnel measurements in both U.S. and Europe, and a preliminary study related to the effects of an azimuthally-periodic array of roughness elements located near the sonic location over the nose tip. The current paper provides an overview of the collaborative work including both theoretical analysis and experimental measurements. The layout of the paper is as follows. First, experimental transition measurements over blunt cones at hypersonic freestream speeds from the literature are summarized and compared in Section II. In Section III, we apply the transient growth analysis to hypersonic blunt cones for selected flow conditions that match the experimental studies relevant to the NATO effort. ${ }^{2,25}$ Those results were used to determine the azimuthal spacing between roughness elements for the experimental measurements of roughness effects within the transition reversal regime. Section IV outlines the preliminary findings from that experiment, while the summary and conclusions are presented in Section V.

\section{Overview of Transition Measurements over Blunt Cones}

The effect of nosetip bluntness on boundary-layer transition is often assessed by plotting the transition Reynolds number as a function of the nosetip radius Reynolds number where both Reynolds numbers are based on the freestream conditions. Figure 1(a) presents the Reynolds number at the start of transition $\left(R e_{\xi_{T}}\right)$ as a function of the nosetip Reynolds number $\left(R e_{R_{N}}\right)$ for the experiments by Stetson ${ }^{2}$ in the Air Force Research Laboratory (AFRL) Mach 6 high-Reynolds-number facility and by Aleksandrova et al. ${ }^{26}$ in the Central Aerohydrodynamic Institute (TsAGI) UT-1M Ludwieg tube. Both data sets are at a nominal freestream Mach number of 6 on straight $8^{\circ}$ half-angle cones. The data from Stetson displays two distinct regions referred to as "small bluntness" where the transition location moves downstream with increased bluntness, and "large bluntness" where the transition location rapidly moves upstream. The data from Aleksandrova et al. ${ }^{26}$ (indicated by star symbols) has a positive slope (small bluntness behavior) up to a critical $R e_{R_{N}}$ of $1.3 \times 10^{5}$. Beyond this critical value, the transition appears to depend on uncontrolled disturbances due to nosetip roughness. In the critical region, groups of points clustered by nosetip radii of 3 , 4, 5, 12, and $14 \mathrm{~mm}$ exhibit a decrease in the transition Reynolds number with an increasing nosetip Reynolds number, which is indicative of transition reversal. With the exception of the sharp and the $0.5 \mathrm{~mm}$ nosetips that consist of steel inserts, the cone model and nosetip inserts were made of AG-4 composite material. The roughness height of the AG-4 material was not specified by the authors, but is expected to be rougher than polished steel. The shape of the transition front, which was quantified using temperature-sensitive paint, revealed turbulent wedges at Reynolds numbers above the critical value. The authors attribute the formation of such wedges to the presence of uncontrolled nosetip roughness. The experiments from Aleksandrova et al. ${ }^{26}$ illustrate that surface roughness has a significant effect on the emergence of the transition reversal. In Stetson's Mach 6 experiments, ${ }^{2}$ the nominal model surface finish had a root-mean-square (rms) value of 15 $\mu$ in. $(0.38 \mu \mathrm{m})$ and blunt nosetips were polished before each run. The polished nosetips most likely explain why the small bluntness behavior was extended to nosetip Reynolds numbers slightly above $9.0 \times 10^{5}$ in Stetson's experiments. The sensitivity of frustum transition to roughness at high nosetip Reynolds numbers was investigated by Stetson by adding $45--50 \mu \mathrm{in}$. $(1.14--1.27 \mu \mathrm{m})$ rms roughness on the $0.6 \mathrm{in}$. (15.2 $\mathrm{mm})$ nosetip. The added roughness caused early frustum transition.

Figure 1(b) presents the Reynolds number at the start of transition as a function of the nosetip Reynolds number for three sets of experiments at nominal freestream Mach numbers between 9 and 10 on straight slender cones. The plot includes the experiments of Stetson ${ }^{2}$ in Tunnel F at Mach 9 on a $7^{\circ}$ half-angle cone, of Marineau et al. ${ }^{25}$ in Tunnel 9 at Mach 10 on a $7^{\circ}$ half-angle cone, and that of Softley et al. ${ }^{27,28}$ 
in the G.E. shock tunnel on a $5^{\circ}$ half-angle cone. As seen at Mach 6, the Mach 10 data also displays the small bluntness and large bluntness regions. The boundary between the large bluntness and small bluntness occurs at varying Reynolds numbers of approximately $1.2 \times 10^{5}, 3.9 \times 10^{5}$, and $9.0 \times 10^{5}$, respectively, for Softley, Marineau, and Stetson. The reason for the variation in the critical Reynolds numbers among the three data sets is not clear, since the respective experiments report similar surface finishes of 30,32 , and $40 \mu$ in. $(0.76,0.81$, and $1.02 \mu \mathrm{m})$. Just like Stetson, Softley and Marineau also polished the nosetip prior to each run, but from the results it appears that the polished surface finish might have been smoother in Stetson's experiment.

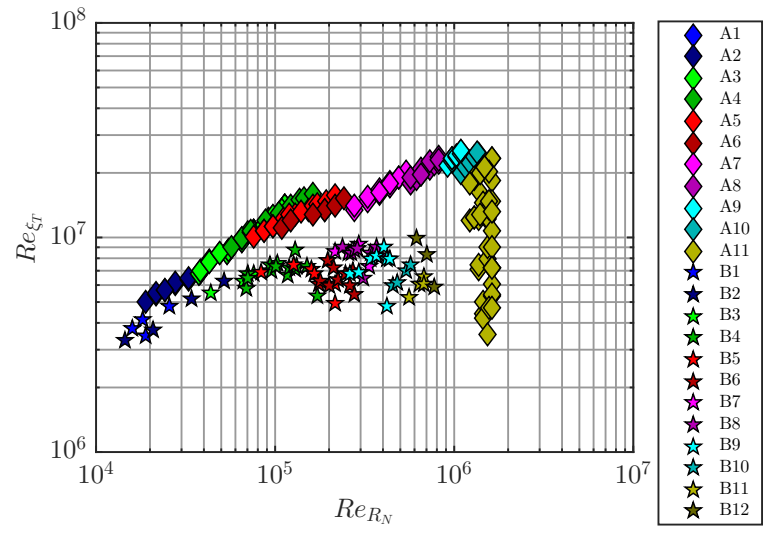

(a) Mach 6. Exp. A from Stetson ${ }^{2}$ with $R_{N}=\operatorname{sharp~(A1),~}$ 0.5 (A2), 1.0 (A3), 1.5 (A4), 2.0 (A5), 2.5 (A6), 5.1 (A7), 7.6 (A8), 10.2 (A9), 12.7 (A10), and $15.2 \mathrm{~mm}$ (A11); Exp. B from Aleksandrova et al. ${ }^{26}$ with $R_{N}=0.5(\mathrm{~B} 1), 1$ (B2), 2 (B3), 3 (B4), 4 (B5), 5 (B6), 6 (B7), 7 (B8), 8 (B9), 10 (B10), 12 (B11), and $14 \mathrm{~mm}(\mathrm{~B} 12)$.

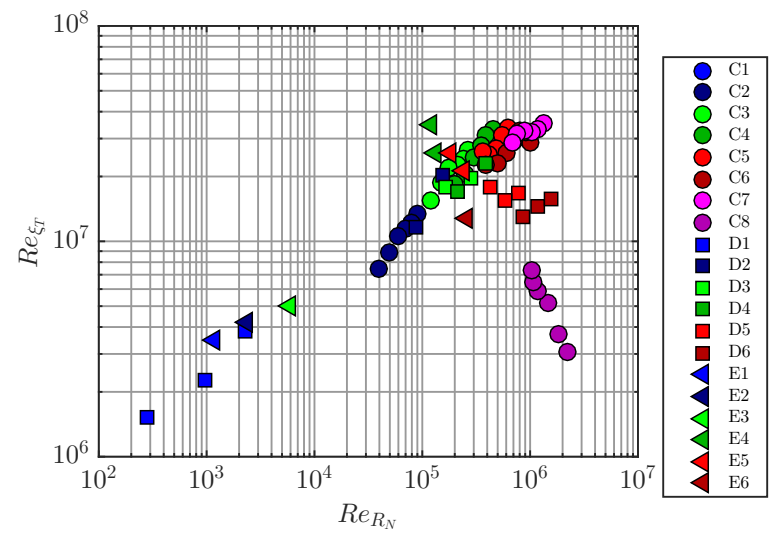

(b) Mach 9 to 10. Exp. C from Stetson ${ }^{2}$ with $R_{N}=$ sharp (C1), 1.5 (C2), 4.5 (C3), 7.5 (C4), 10.5 (C5), 14.0 (C6), 22.5 (C7), 55.4 (C8); Exp. D from Marineau et al. ${ }^{25}$ with $R_{N}=0.15$ (D1), 5.1 (D2), 9.5 (D3), 12.7 (D4), 25.4 (D5), and $50.1 \mathrm{~mm}$ (D6); Exp. E from Softley et al. ${ }^{27,28}$ with $R_{N}=0.25$ (E1), 0.51 (E2), 1.3 (E3), 12.7 (E4), 19.1 (E5), and $25.4 \mathrm{~mm}(\mathrm{E} 6)$

Figure 1. Transition Reynolds number based on freestream as a function of the nose Reynolds number at (a) Mach 6 and (b) Mach 9 to 10 which illustrates the effect of bluntness and the transition reversal.

As noted by Stetson, ${ }^{29}$ the use of "small bluntness" and "large bluntness" when discussing nosetip bluntness effects can be misleading, as the bluntness effect is related not only to the physical dimensions of the nosetip, but also to where transition occurs with respect to the nosetip. As discussed by Muir and Trujillo, ${ }^{30}$ the nosetip Reynolds number does not properly account for the various effects of the nosetip radius and unit Reynolds numbers. Stetson and Rushton ${ }^{31}$ introduced the entropy-swallowing length as a parameter to relate the transition location to nosetip bluntness effects. Using Rotta's correlation, ${ }^{32}$ the entropy-swallowing length is found to be a function of $\left(R e_{\infty}\right)^{1 / 3}$ and $\left(R_{N}\right)^{4 / 3}$. One disadvantage of the entropy-swallowing length as a correlation parameter is that it cannot be easily defined for arbitrary geometries. Moreover, the blunt cone data is usually correlated by normalizing the transition locations (location or Reynolds numbers) on blunt cones by the transition length on sharp cones in order to remove unit Reynolds number effects. Such an approach cannot be extended to arbitrary geometries.

Boundary-layer stability calculations have tried to explain the transition behavior in hypersonic blunt cones. The effect of bluntness on the second-mode instability was first investigated by Malik et al. ${ }^{33}$ and Herbert \& Esfahanian ${ }^{34}$ and more recently by Marineau et al. ${ }^{25,35}$ and Jewell and Kimmel. ${ }^{36}$ These recent studies include parabolized stability equation (PSE) analysis of the historical Stetson Mach 6 and Mach 9 blunt cone experiments using the STABL software suite. ${ }^{37}$ The stability analyses agree that the transition reversal cannot be predicted by only considering Mack's second-mode instability mechanism. This is because increased bluntness stabilizes the second mode by moving the neutral point downstream due to local edge Mach number and local unit Reynolds number reductions within the entropy layer. This implies that the transition location based on Mack's second-mode amplification keeps moving downstream and eventually transition does not occur as the bluntness increases. In addition, the boundary-layer stability studies found that the first mode is also not destabilized by bluntness, so that it cannot be responsible for the transition reversal.

Even if transition reversal cannot be predicted with linear stability computations of the first and second modes, an approach combining the measurements and computations data will still be useful to evaluate 
where and when the transition process is no longer dominated by the second mode. Figure 2 presents a compilation of second-mode transition $N$-factors $N_{T}$ as a function of the nosetip Reynolds number $\operatorname{Re}_{R_{N}}$. For values of $R e_{R_{N}}$ below $1 \times 10^{3}, N_{T}$ increases with $R e_{R_{N}}$ as a result of the increase in the unit Reynolds number. This behavior can be linked to an increase in the critical second-mode frequency $f_{T}$, which implies a lower tunnel noise content near $f_{T}$. This effect, first discussed by Marineau et al. ${ }^{25}$ and Marineau, ${ }^{35}$ has recently been modeled by Balakumar and $\mathrm{Chou}^{38}$ with direct numerical simulations (DNS) of the Tunnel 9 Mach 10 experiments. The approach includes the measured freestream noise spectrum and an empirical correlation (see Marineau et al. ${ }^{25}$ ) to determine the breakdown amplitude of the second mode. The sharp cone nosetip radius was not specified in either Stetson Mach 6 or Mach 9 experiments. To include these sharp cone data points in Fig. 2, the sharp nosetips were assumed to have the same radius as the sharp Tunnel 9 cone. A variation in the sharp cone nosetip radius does not change the trends, as it simply shifts the point left or right. For $\operatorname{Re}_{R_{N}}$ between approximately $4 \times 10^{4}$ and $1 \times 10^{5}$, a steep decrease in $N_{T}$ with $R e_{R_{N}}$ is observed. Somewhere in this region, the transition process appears to not be dominated by second-mode amplification. Note that the departure from second-mode-dominated transition occurs prior to transition reversal. The decrease of $N_{T}$ with bluntness was discussed by Marineau ${ }^{35}$ and attributed to a decrease of the second-mode breakdown amplitudes and to an increase in the initial amplitudes. The decrease in the breakdown amplitudes is linked to the lower edge Mach number whereas the increase in the initial amplitudes is related to the decrease in the critical second-mode frequency $f_{T}$ as well as an increase in the receptivity coefficient with $R e_{R_{N}}$. For these conditions, the measured and estimated transition $N$-factors and the estimated receptivity coefficients are shown in Figs. 3(a) and 3(b), respectively.

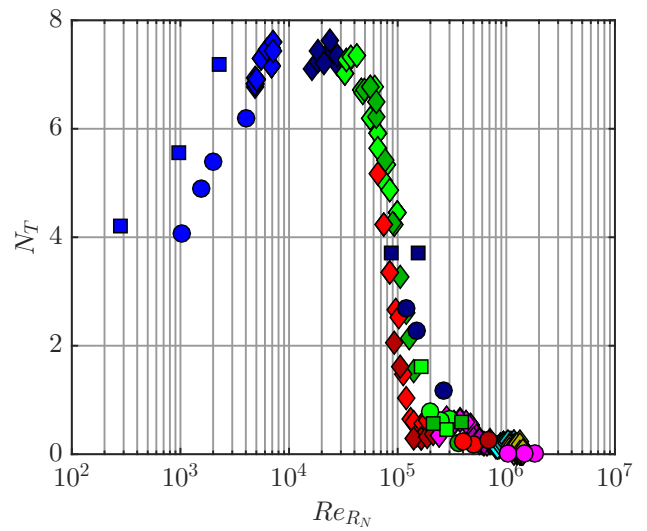

Figure 2. Computed second-mode $N$-factors at the experimental transition location (start of transition) as a function of the nosetip Reynolds number for the Mach 6 experiments of Stetson ${ }^{2}$ from Ref. 36 (diamonds), Mach 9 experiments of Stetson $^{2}$ from Ref. 35 (circles) and Mach 10 experiments of Marineau et al. ${ }^{25}$ (squares). The same symbols and colors as in Fig. 1 are used to indicate the nosetip radius.

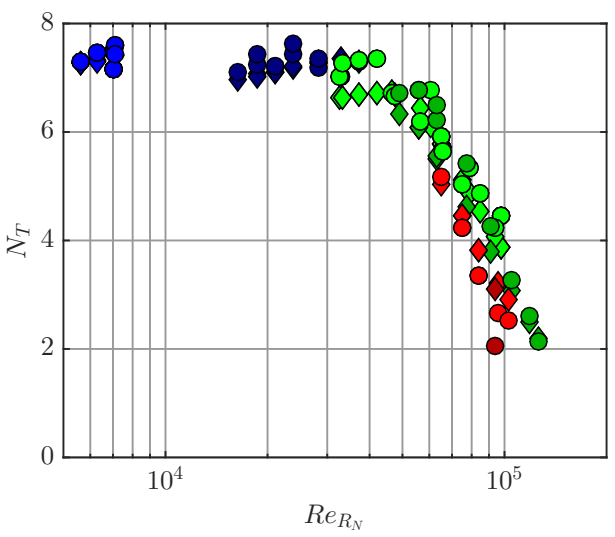

(a) Measured (diamonds) and estimated (circles) transition $N$-factors as a function of the nosetip Reynolds number.

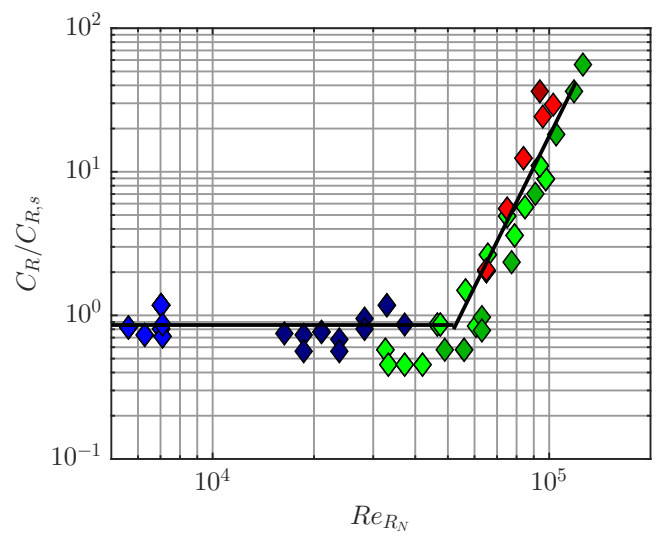

(b) Estimated receptivity coefficients $C_{R}$ normalized by the average sharp cone receptivity coefficient $C_{R, s}$.

Figure 3. Effect of bluntness on receptivity and transition $N$-factors for Stetson blunt cone experiments at Mach 6 . 
In contrast to Stetson's blunt cone experiments, which measured just the transition location, Marineau et al. $^{25}$ also measured the boundary-layer instabilities by using a large number of high-frequency response pressure sensors $\left(\mathrm{PCB}^{\circledR}-132\right)$. These measurements captured the evolution of the pressure fluctuations over the surface of the cone. Figure 4 presents a map of the logarithm of the pressure power spectral density $(\log (\mathrm{PSD}))$ at $R e_{\infty} \approx 17 \times 10^{6} \mathrm{~m}^{-1}$ for $R_{N}=0.15,5.1,9.5,12.7,25.4$, and $50.8 \mathrm{~mm}$. For $R_{N} \geq 5.1 \mathrm{~mm}$, the transition occurs before the entropy layer is swallowed. This leads to a significant reduction of the edge Mach number compared to the sharp cone case. For the $5.1 \mathrm{~mm}$ nosetip in Fig. 4(b), the edge Mach number varies from 4.4 to 4.8 between the neutral point and the start of transition. The bluntness significantly delays the appearance of the second-mode waves and increases the distances over which growth and breakdown occur. As a result of these two factors, the transition location is moved further downstream on the blunt cone (from $0.25 \mathrm{~m}$ on the sharp cone to $0.68 \mathrm{~m}$ on the $5.1 \mathrm{~mm}$ cone, based on heat transfer measurements). In addition, the second-mode frequencies are significantly lower on the blunt cone due to the increased boundary-layer thickness. As the nosetip radius increases from $5.1 \mathrm{~mm}$ to $9.5 \mathrm{~mm}$, the transition location moves further downstream and the unstable second-mode frequencies are further decreased. The increase from $9.5 \mathrm{~mm}$ to $12.7 \mathrm{~mm}$ has a minor effect on the transition location, which indicates that reversal is near. For the 12.7 $\mathrm{mm}$ nosetip, the start of transition occurs prior to significant growth of the second mode. This indicates that transition was not initiated by the second-mode instability. However, further downstream within the transitional region, the second-mode amplitudes keep increasing up to the downstream end of the cone. The 25.4 and $50.8 \mathrm{~mm}$ nosetips are in the reversal regime as the transition location has moved upstream compared to those at the smaller radii. In addition, transition occurs before the appearance of second-mode waves. This makes sense, as the edge Mach number at the start of transition for the $25.4 \mathrm{~mm}$ and $50.8 \mathrm{~mm}$ radii are 3.3 and 3.2 respectively, which are too low for second-mode growth. The results for $25.4 \mathrm{~mm}$ and $50.8 \mathrm{~mm}$ nosetips also reveal that the mechanism responsible for the transition reversal has a weak pressure signature, as no significant variation in the pressure PSD is found.

The use of fast-response heat flux sensors can help to characterize the transition mechanisms on blunt cones. For instance, time-resolved heat transfer measurements performed at Mach 9 by Zanchetta ${ }^{39}$ in the Imperial College Gun Tunnel on a $5^{\circ}$ half-angle cone revealed that in the reversal regime, transitional events are formed in the near-nose region and convect downstream. The formation frequency of the events was linked to the severity of the roughness environment. In certain cases, second-mode instability waves and these transition events were occurring concurrently; and the experiments indicated that the second mode was responsible for the completion of transition. Recent laser-induced-fluorescence-based (LIF) schlieren measurements from Grossir et al. ${ }^{40}$ on a blunt $7^{\circ}$ half-angle cone at Mach 10 in the von Karman Institute Longshot hypersonic wind-tunnel revealed disturbances with shapes quite different from the usual secondmode rope structures. The disturbances that extend above the edge of the boundary layer are seen in Fig. 5 for the $4.75 \mathrm{~mm}$ nosetip radius. These disturbances, that were not present on schlieren images for sharper cones, could be a manifestation of the blunt cone transition mechanism leading to the heat transfer fluctuation measured by Zanchetta. ${ }^{39}$

In summary, the experimental and numerical studies agree that frustum transition in the reversal regime cannot be accounted for via linear, modal instability analysis. Furthermore, the experimental observations agree that frustum transition on large bluntness cones is highly sensitive to wall roughness and appears to be dominated by disturbances that originate in the vicinity of the nosetip. Therefore, roughness-induced transient growth emerges as the primary candidate for the experimentally-observed trend in laminar-turbulent transition.

\section{Transient Growth Analysis of Hypersonic Blunt Cones}

This section presents the transient growth analysis of blunt circular cones with conditions selected to match a subset of the configurations from the experiments conducted by Stetson ${ }^{2}$ in the Air Force Research Laboratory (AFRL) Mach 6 High Reynolds Number facility and by Marineau ${ }^{25}$ in the Arnold Engineering Development Complex (AEDC) Tunnel 9 at Mach 10. Modal instability analysis for the AFRL configurations has been already performed by Jewell \& Kimmel, ${ }^{36}$ and Marineau ${ }^{25}$ has described similar analysis for the AEDC configurations. They found that both first-mode and Mack-mode waves were either damped or weakly unstable for the present configurations; and therefore, transition reversal cannot be predicted with the modal analysis. Another modal instability mechanism that might play a role in the transition reversal is the entropy-layer instability. ${ }^{41,42}$ However, although not shown here, our analysis did not revealed any 


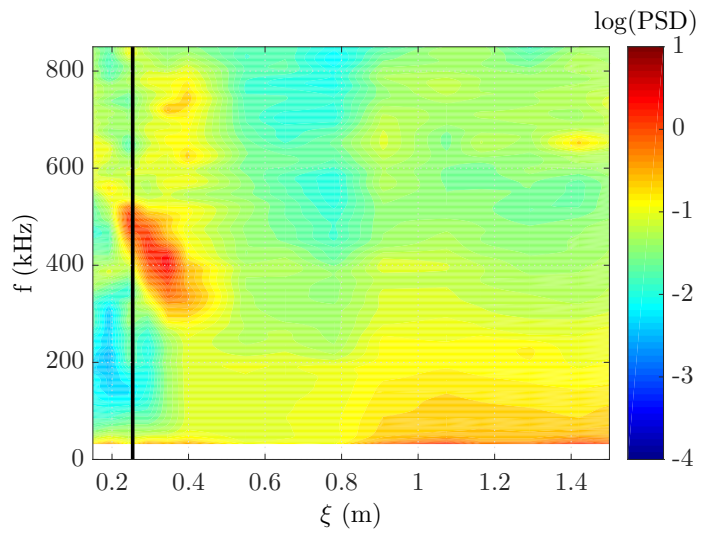

(a) $R_{N}=0.15 \mathrm{~mm}\left(\xi_{T}=0.254 \mathrm{~m}\right)$

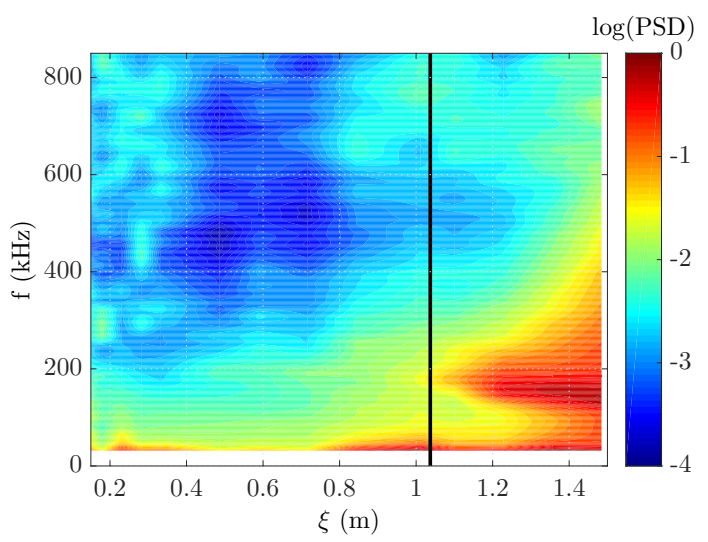

(c) $R_{N}=9.5 \mathrm{~mm}\left(\xi_{T}=1.037 \mathrm{~m}\right)$

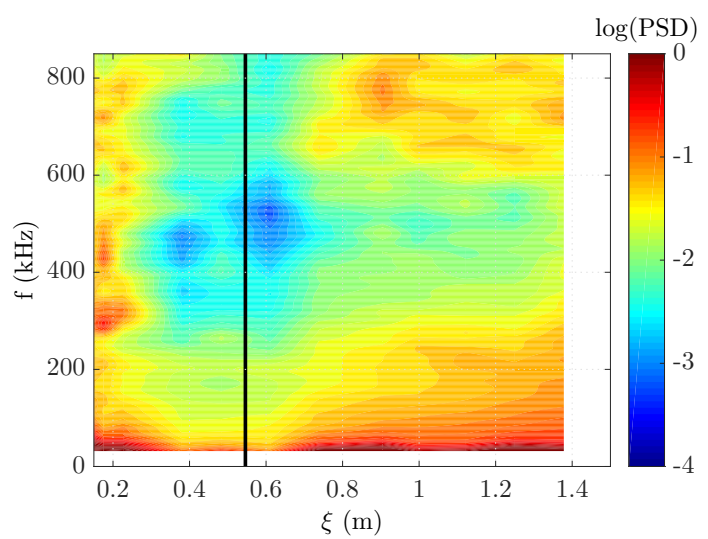

(e) $R_{N}=25.4 \mathrm{~mm}\left(\xi_{T}=0.546 \mathrm{~m}\right)$

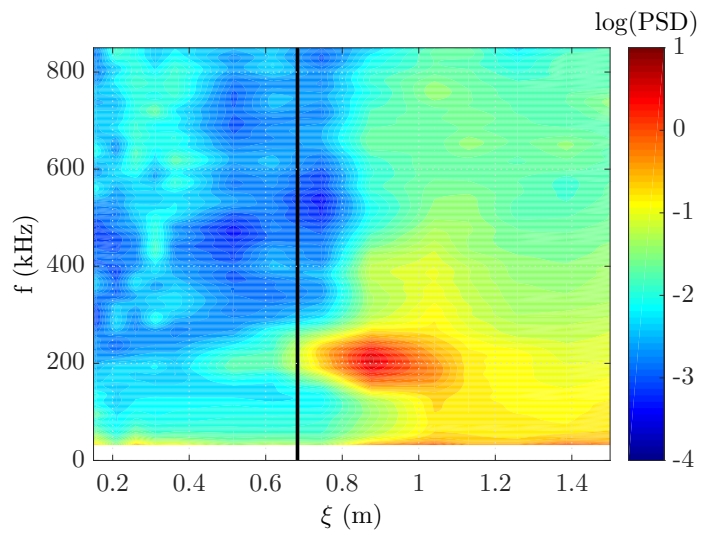

(b) $R_{N}=5.1 \mathrm{~mm}\left(\xi_{T}=0.683 \mathrm{~m}\right)$

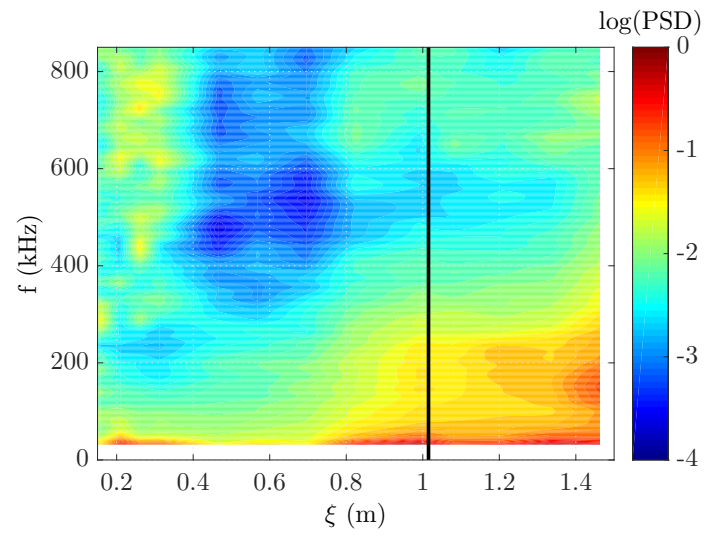

(d) $R_{N}=12.7 \mathrm{~mm}\left(\xi_{T}=1.015 \mathrm{~m}\right)$

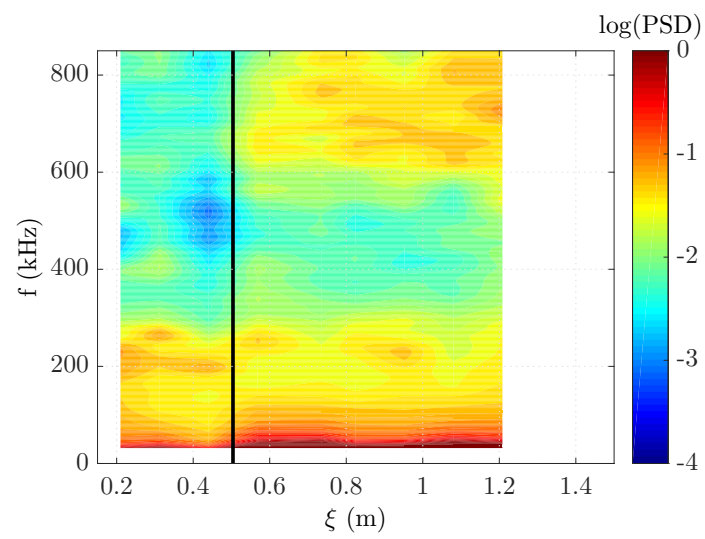

(f) $R_{N}=50.8 \mathrm{~mm}\left(\xi_{T}=0.504 \mathrm{~m}\right)$

Figure 4. Contour map of the logarithm of the pressure power spectral density $\log (\mathrm{PSD})$ for sharp and blunt cones at $\operatorname{Re}_{\infty} \approx 17 \times 10^{6} \mathbf{m}^{-1}$ at Mach 10 in Tunnel 9. The vertical, black line denotes the measured transition location. 


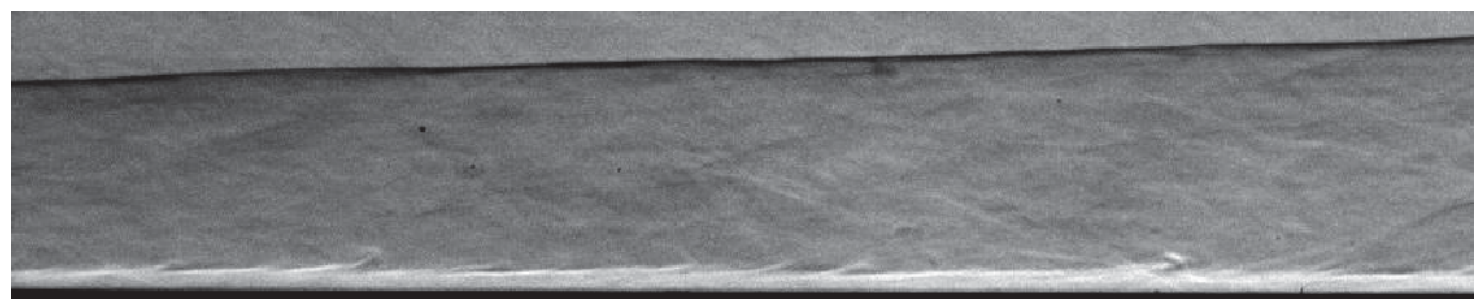

Figure 5. LIF-based schlieren flow visualization on $4.75 \mathrm{~mm}$ radius nosetip $7^{\circ}$ half-angle cone in $\mathrm{VKI}$ at $M_{\infty}=11.9$ and $R e_{\infty}=11.6 \times 10^{6} \mathbf{m}^{-1}$. Fields of view extend from $x=625 \mathrm{~mm}$ until the end of the cone at $806 \mathrm{~mm}$. Disturbance extending past the boundary-layer thickness are visible.

substantially amplified entropy-layer modes for the configurations of interest. Therefore, the transient growth mechanism is investigated as a potential cause for the transition reversal. First, the basic state solutions are presented in subsection III.A. Second, the transient growth theory is briefly introduced in subsection III.B. Then, a detailed transient growth analysis of the selected blunt cones configurations is presented in subsection III.C.

\section{III.A. Laminar Boundary Layer over Blunt Cones}

The basic states used in the present analysis correspond to the laminar boundary layer flow over the selected blunt cone configurations. The laminar boundary layer flows were computed by Jewell \& Kimmel ${ }^{36}$ and Marineau $^{25}$ with reacting, axisymmetric Navier-Stokes equations on a structured grid. The solver was a version of the NASA data parallel-line relaxation (DPLR) code, ${ }^{43}$ that is included as part of the STABL-2D software suite, as described by Johnson ${ }^{44}$ and Johnson et al. ${ }^{45}$ This flow solver employs a second-orderaccurate finite-volume formulation. The inviscid fluxes are based on the modified Steger-Warming flux vector splitting method with a monotonic upstream-centered scheme for the conservation laws (MUSCL) limiter. The time integration method is the implicit, first-order data parallel line relaxation (DPLR) method. Additional details about the basic state solution and the grid convergence study are given by Jewell \& Kimmel $^{36}$ for the AFRL configurations and by Marineau ${ }^{25}$ for the AEDC configurations.

\section{III.A.1. AFRL Configurations}

The AFRL Mach 6 facility operates at stagnation pressures $p_{0}$ from 700 to 2100 psi (4.83 to $14.5 \mathrm{MPa}$ ). The working fluid is air and is treated as ideal gas because of the relatively low temperature and pressure. The blunt cones used in the experiments have a half-angle of $8^{\circ}$ and a base radius of $2.0 \mathrm{in}$. (0.0508 m). A total of 196 experiments encompassing 108 unique conditions comprised the Stetson ${ }^{2}$ Mach 6 results. Table 1 shows the details of the four configurations selected for the present analysis. The present analysis uses the $7^{\circ}$ half-angle variable-bluntness cone that is currently used in the experiments in the AFRL Mach 6 facility. The thermal wall condition is isothermal with a constant wall temperature equal to $\bar{T}_{w}=300.0 \mathrm{~K}$.

Table 1. Details of the four AFRL configurations used in the present study. The wall temperature is $\bar{T}_{w}=300 \mathrm{~K}$.

\begin{tabular}{ccccc}
$R_{N}(\mathrm{~mm})$ & $R e_{\infty}\left(\times 10^{6} \mathrm{~m}^{-1}\right)$ & $M_{\infty}$ & $\bar{T}_{\infty}$ & $\bar{T}_{w} / \bar{T}_{w, a d}$ \\
\hline 5.080 & 91.4 & 5.9 & 76.74 & 0.57 \\
15.24 & 91.4 & 5.9 & 76.74 & 0.57 \\
15.24 & 60.9 & 5.9 & 76.74 & 0.57 \\
15.24 & 30.5 & 5.9 & 76.74 & 0.57 \\
\hline
\end{tabular}

The streamwise evolution of the boundary layer thickness $\delta_{h}$ and edge Mach number $M_{e}$ is plotted in Fig. 6. The boundary-layer edge, $\eta_{e}=\delta_{h}$, is defined as the wall-normal position where $h_{t} / h_{t, \infty}=0.995$, with $h_{t}$ denoting the total enthalpy, i.e., $h_{t}=h+0.5\left(\bar{u}^{2}+\bar{v}^{2}+\bar{w}^{2}\right)$, where $h$ is the static enthalpy. The evolution of boundary layer thickness $\delta_{h}$ within the nose region and along the entire geometry is shown in Figs. 6(a) and 6(b), respectively. The boundary layer thickness is nearly constant from the stagnation point up to $\phi \approx 30^{\circ}$, which is characteristic of stagnation boundary layer flow. From $\phi \approx 45^{\circ}$, the boundary layer thickness grows monotonically up to the end of the cone, as shown in Fig. 6(b). Figs. 6(a) and 6(b) also show that the boundary layer thickness for the smaller nose radius $\left(R_{N}=9.53 \mathrm{~mm}\right)$ cone is smaller than for 
the larger nose radius $\left(R_{N}=15.24 \mathrm{~mm}\right)$ cone with the same freestream conditions $\left(R e_{\infty}=91.4 \times 10^{6} \mathrm{~m}^{-1}\right)$. Figures 6(c) and 6(d) show the evolution of the edge Mach number in the nose region and in the entire cone, respectively. The edge Mach number is determined by inviscid theory to the leading order, and therefore, the evolution within the nose is nearly coincident for the four configurations (Fig. 6(c)). However, the edge Mach number evolution is clearly distinguishable from the smaller to the larger nose radius cases when it is plotted against the streamwise coordinate for the entire geometry ((Fig. 6(d)). The sonic location, which coincides with the peak of the streamwise mass-flux within the nose, ${ }^{23,24}$ is found at $\phi=41.4^{\circ}$. The edge Mach number remains below $M_{e}=3$ along the entire geometry for the large nose radius cones and only becomes slightly larger than $M_{e}=3$ at the end of the cone for the smaller nose radius case.

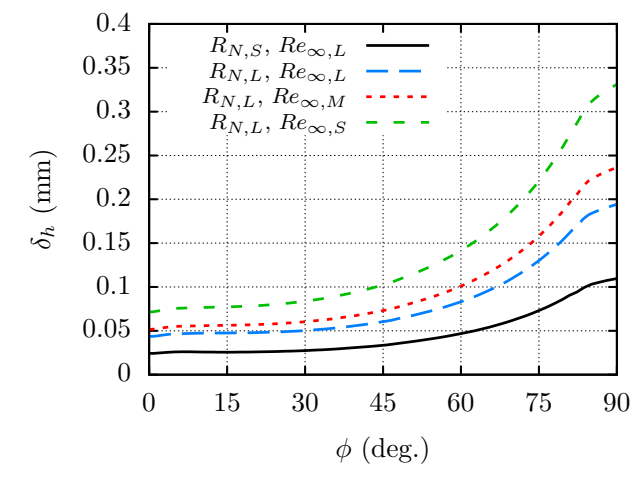

(a) Boundary layer thickness evolution within the nose.

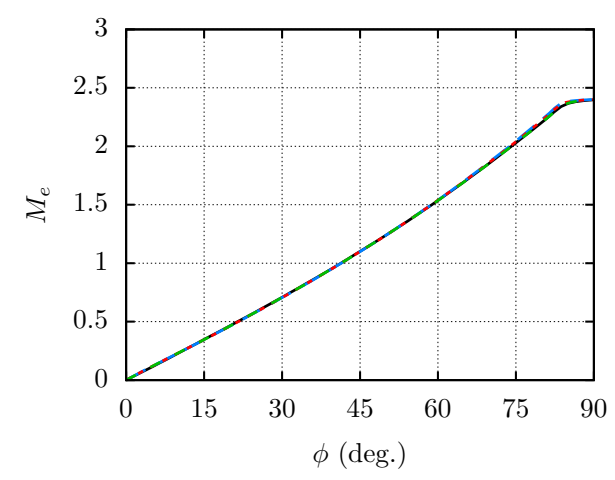

(c) Edge Mach number evolution within the nose.

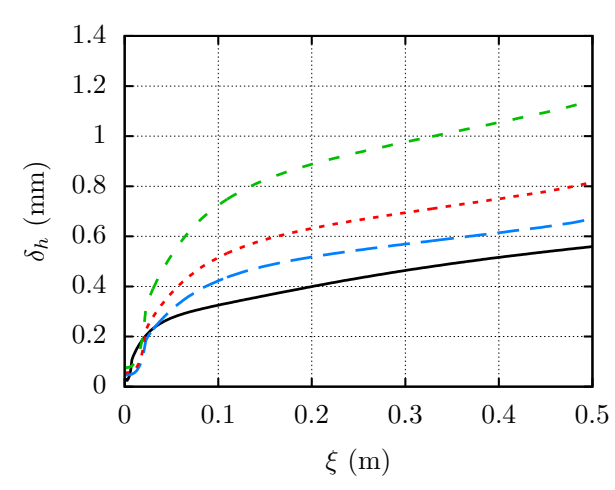

(b) Boundary layer thickness evolution along the cone.

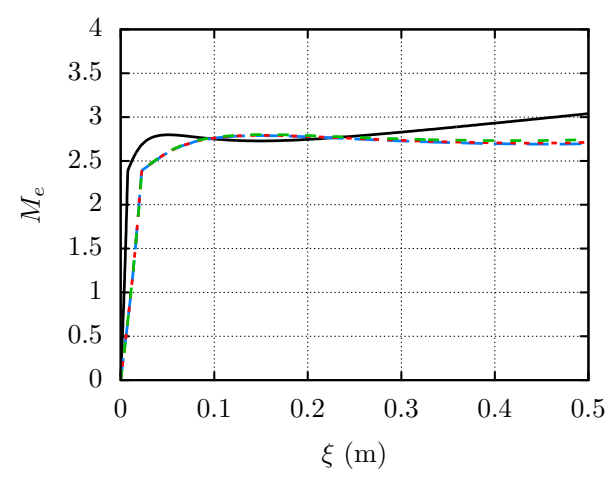

(d) Edge Mach number evolution along the cone.

Figure 6. Streamwise evolution of boundary layer thickness and edge Mach number of the laminar boundary layer flows over the AFRL configurations. The legend refers to $R_{N, S}=5.08 \mathbf{m m}, R_{N, L}=15.24 \mathbf{m m}, \operatorname{Re}_{\infty}, L=91.4 \times 10^{6} \mathbf{m}^{-1}$, $R e_{\infty, M}=60.9 \times 10^{6} \mathbf{m}^{-1}, R e_{\infty, S}=30.5 \times 10^{6} \mathbf{m}^{-1}$.

\section{III.A.2. AEDC Configurations}

The Air Force AEDC Hypervelocity Wind Tunnel No. 9 (Tunnel 9) is a hypersonic, nitrogen gas, blowdown wind tunnel with interchangeable nozzles that allow for testing at Mach numbers of 7, 8, 10, and 14 over a $0.177 \times 10^{6} \mathrm{~m}^{-1}$ to $158.8 \times 10^{6} \mathrm{~m}^{-1}$ unit Reynolds number range. A detailed description of the facility can be found in Ref. 25. The blunt cones used in the experiments of Marineau et al. ${ }^{25}$ had a base diameter of $15 \mathrm{in} .(0.381 \mathrm{~m})$ and interchangeable nose tips with radius of $0.152 \mathrm{~mm}$ to $50.80 \mathrm{~mm}$. The test matrix for the 24 run test program is provided in Ref. 25. The working fluid is nitrogen at a relatively low temperature and pressure. Thus, the effects of chemistry and molecular vibration are omitted from the calculations. The viscosity law used is the Sutherland's law and the heat conductivity is calculated using Eucken's relation. Table 2 shows the details of the four configurations selected for the present analysis. The used thermal wall condition is isothermal with a constant wall temperature equal to $\bar{T}_{w}=300.0 \mathrm{~K}$. The four configurations share a similar freestream unit Reynolds number of $R e_{\infty} \approx 17.5 \times 10^{6} \mathrm{~m}^{-1}$ and a freestream Mach number of $M_{\infty} \approx 9.78$. The nose radius values varies from $R_{N}=9.53 \mathrm{~mm}$ to $R_{N}=50.8 \mathrm{~mm}$.

The boundary layer thickness $\delta_{h}$ and edge Mach number $M_{e}$ for the four configurations are plotted in 
Table 2. Details of the four AEDC configurations used in the present study. The wall temperature is $\bar{T}_{w}=300 \mathrm{~K}$.

\begin{tabular}{ccccc}
$R_{N}(\mathrm{~mm})$ & $R e_{\infty}\left(\mathrm{m}^{-1}\right)$ & $M_{\infty}$ & $\bar{T}_{\infty}$ & $\bar{T}_{w} / \bar{T}_{w, a d}$ \\
\hline 9.530 & $17.6 \times 10^{6}$ & 9.797 & 51.01 & 0.340 \\
12.70 & $17.4 \times 10^{6}$ & 9.795 & 51.17 & 0.339 \\
25.40 & $17.3 \times 10^{6}$ & 9.791 & 51.13 & 0.340 \\
50.80 & $17.6 \times 10^{6}$ & 9.777 & 51.27 & 0.340 \\
\hline
\end{tabular}

Figs. 7(a) and 7(b), respectively. As observed in the comparison of the AFRL configurations of Fig. 6(b), despite the same freestream conditions, the boundary layer thickness in the frustum part of the cone is larger for larger nose radius values. Also, the edge Mach number is larger for the smaller nose radius cases, although remains smaller than $M_{e}=4.5$ for the four configurations.

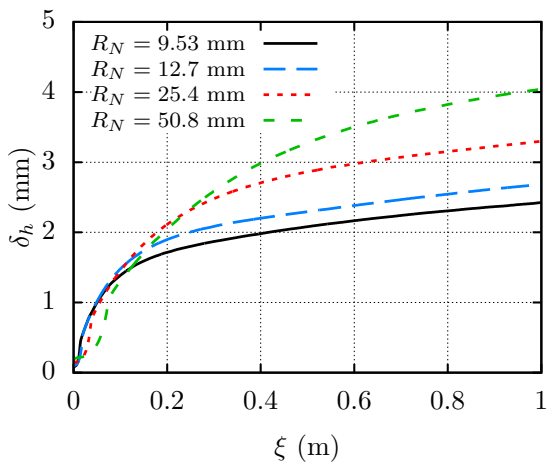

(a) Boundary layer thickness.

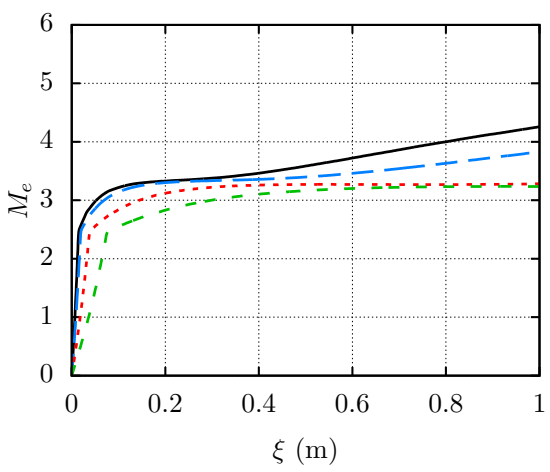

(b) Edge Mach number evolution.

Figure 7. Streamwise evolution of boundary layer thickness and edge Mach number of the laminar boundary layer flows over the AEDC configurations.

\section{III.B. Transient Growth Theory}

Transient growth analysis is performed using the linear PSE as explained in the literature. ${ }^{18,23,46-48}$ The analysis focuses on stationary perturbations because, despite of the speed regime, they experience the largest transient growth. For completeness, the present section outlines the methodology, which bears strong similarities with the optimization approach based on the linearized boundary layer equations. ${ }^{17,22,49}$ The advantage of the PSE-based formulation is that it is also applicable to more complex base flows where the flow evolves along the streamwise direction and the boundary layer approximation may not hold. The PSE approach can also be easily extended to unsteady disturbances. While infinite Reynolds number asymptotic results cannot be directly computed using this technique, good agreement is achieved between the two methodologies for incompressible and compressible regimes as shown by Paredes et al. ${ }^{18}$

In the PSE context, stationary perturbations have the form

$$
\tilde{\mathbf{q}}(\xi, \eta, \zeta)=\hat{\mathbf{q}}(\xi, \eta) \exp [\mathrm{i}(m \zeta)]+\text { c.c. }
$$

where c.c. denotes complex conjugate. The suitably nondimensionalized, orthogonal, curvilinear coordinate system $(\xi, \eta, \zeta)$ denotes streamwise, wall-normal, and azimuthal coordinates and $(u, v, w)$ represent the corresponding velocity components. The azimuthal wavenumber is denoted as $m$. Density and temperature are denoted by $\rho$ and $T$. The Cartesian coordinates are represented by $(x, y, z)$. The vector of perturbation fluid variables is $\tilde{\mathbf{q}}(\xi, \eta, \zeta, t)=(\tilde{\rho}, \tilde{u}, \tilde{v}, \tilde{w}, \tilde{T})^{T}$ and the vector of amplitude functions is $\hat{\mathbf{q}}(\xi, \eta)=(\hat{\rho}, \hat{u}, \hat{v}, \hat{w}, \hat{T})^{T}$. The vector of basic state variables is $\overline{\mathbf{q}}(\xi, \eta)=(\bar{\rho}, \bar{u}, \bar{v}, \bar{w}, \bar{T})^{T}$.

Upon introduction of the perturbation form (1) into the linearized NS equations together with the assumption of a slow streamwise dependence of the basic state and the amplitude functions, thus neglecting the viscous derivatives in $\xi$, the PSE are recovered as follows

$$
\mathbf{L} \hat{\mathbf{q}}(\xi, \eta)=\left(\mathbf{A}+\mathbf{B} \frac{\partial}{\partial \eta}+\mathbf{C} \frac{\partial^{2}}{\partial \eta^{2}}+\mathbf{D} \frac{1}{h_{1}} \frac{\partial}{\partial \xi}\right) \hat{\mathbf{q}}(\xi, \eta)=0 .
$$


The linear operators $\mathbf{A}, \mathbf{B}, \mathbf{C}$ and $\mathbf{D}$ are given by Pralits et al. ${ }^{46}$ and $h_{1}$ is the metric factor associated with the streamwise curvature. The system of Eqs. (2) is not fully parabolic due to the term $\partial \hat{p} / \partial \xi$ in the streamwise momentum equation. ${ }^{50-53}$ However, for the purely stationary disturbances of interest in this work, this term can be dropped from the equations as justified by Refs. 47,54, which found that the term, $\partial \hat{p} / \partial \xi$, is of higher order for transient growth problems, and can be neglected without any loss of accuracy.

The optimal initial disturbance, $\tilde{\mathbf{q}}_{0}$, is defined as the initial (i.e., inflow) condition at $\xi_{0}$ that maximizes the objective function, $J$, which is defined as a measure of disturbance growth over a specified interval $\left[\xi_{0}, \xi_{1}\right]$. The definitions used in the present study correspond to the outlet energy gain $J=G^{\text {out }}$ and mean energy gain $J=G^{\text {mean }}$ and are defined as

$$
\begin{gathered}
G^{\text {out }}=\frac{E\left(\xi_{1}\right)}{E\left(\xi_{0}\right)}, \\
G^{\text {mean }} \frac{1}{\xi_{1}-\xi_{0}} \frac{\int_{\xi_{0}}^{\xi_{1}} E\left(\xi^{\prime}\right) d \xi^{\prime}}{E\left(\xi_{0}\right)},
\end{gathered}
$$

where $E$ denotes the energy norm of $\tilde{\mathbf{q}}$. The energy norm is defined as

$$
E(\xi)=\int_{\eta} \hat{\mathbf{q}}(\xi, \eta)^{H} \mathbf{M}_{E} \hat{\mathbf{q}}(\xi, \eta) h_{1} h_{3} \mathrm{~d} \eta
$$

where $h_{3}$ is the metric factor associated with the azimuthal curvature, $\mathbf{M}_{E}$ is the energy weight matrix and the superscript $H$ denotes conjugate transpose. The selection of $J=G^{\text {out }}$ corresponds to the "outlet energy gain" that is commonly used in studies of the optimal-perturbation problem. ${ }^{22,49}$ The selection of $J=G^{\text {mean }}$ defines the "mean energy gain" and corresponds to the optimization of the mean energy. This latter definition accounts for a possible overshoot in the disturbance energy evolution that are not accounted for by the former definition and is found to be present in hypersonic blunt forebodies, ${ }^{23,24}$ as well as in the nosetip of blunt cones as documented in what follows.

The choice of the energy norm is known to influence the optimal initial perturbation as well as the magnitude of energy amplification. ${ }^{17,48,55}$ Here, we use the positive-definite energy norm derived by Mack ${ }^{56}$ and used by Hanifi et al. ${ }^{57}$ for transient growth calculations, which is defined by

$$
\mathbf{M}_{E}=\operatorname{diag}\left[\frac{\bar{T}(\xi, \eta)}{\gamma \bar{\rho}(\xi, \eta) M^{2}}, \bar{\rho}(\xi, \eta), \bar{\rho}(\xi, \eta), \bar{\rho}(\xi, \eta), \frac{\bar{\rho}(\xi, \eta)}{\gamma(\gamma-1) \bar{T}(\xi, \eta) M^{2}}\right] .
$$

Additionally, the kinetic energy norm is also used for optimization in this paper. The kinetic energy of a perturbation is defined by

$$
K(\xi)=\int_{\eta} \hat{\mathbf{q}}(\xi, \eta)^{H} \mathbf{M}_{K} \hat{\mathbf{q}}(\xi, \eta) h_{1} h_{3} \mathrm{~d} \eta
$$

where

$$
\mathbf{M}_{K}=\operatorname{diag}[0, \bar{\rho}(\xi, \eta), \bar{\rho}(\xi, \eta), \bar{\rho}(\xi, \eta), 0] .
$$

To differentiate when the total energy norm $E$ or the kinetic energy norm $K$ are used, a corresponding subscript is added to the energy gain, resulting in four options for the objective function: $G_{E}^{\text {out }}, G_{E}^{\text {mean }}$, $G_{K}^{\text {out }}$, and $G_{K}^{\text {mean }}$. In the present study, the transient growth amplification is also expressed in terms of the logarithmic amplification ratio, the so-called $N$-factor, based on the total energy norm, which is defined as

$$
N_{E}=\frac{1}{2} \ln \left(\mathrm{G}_{\mathrm{E}}^{\text {out }}\right) .
$$

The variational formulation of the problem to determine the maximum of the objective functional $J$ leads to an optimality system, ${ }^{18}$ which is solved in an iterative manner, starting from a random solution at $\xi_{0}$ that must satisfy the boundary conditions. The PSE, L $\tilde{\mathbf{q}}=0$, are used to integrate $\tilde{\mathbf{q}}$ up to $\xi_{1}$, where the final optimality condition is used to obtain the initial condition for the backward adjoint PSE integration, $\mathbf{L}^{\dagger} \tilde{\mathbf{q}}^{\dagger}=c_{\text {mean }} F(\tilde{\mathbf{q}})$, where $c_{\text {mean }}=0$ for the outlet energy gain optimization and $c_{\text {mean }}=1$ for the mean energy gain optimization, and $F(\tilde{\mathbf{q}})$ is a function of the direct solution. ${ }^{46}$ At $\xi_{0}$, the adjoint solution is used to calculate the new initial condition for the forward PSE integration with the initial optimality condition. The iterative procedure finishes when the value of $G$ has converged up to a certain tolerance, which was set to $10^{-4}$ in the present computations. 
Nonuniform stable high-order finite-difference schemes ${ }^{58,59}$ of sixth order are used for discretization of the PSE along the wall-normal coordinate. The discretized PSE are integrated along the streamwise coordinate by using second-order backward differentiation. The number of discretization points in both directions was varied in selected cases to ensure convergence of the optimal gain predictions. The wall-normal direction was discretized using $N_{y}=161$, with the nodes being clustered toward the wall. ${ }^{59}$ No-slip, isothermal boundary conditions are used at the wall, i.e., $\hat{u}=\hat{v}=\hat{w}=\hat{T}=0$. The amplitude functions are forced to decay at the farfield boundary by imposing the Dirichlet conditions $\hat{\rho}=\hat{u}=\hat{w}=\hat{T}=0$, unless otherwise stated. The farfield boundary coordinate is set just below the shock layer. Verification of the present optimal growth module against available transient growth results from the literature is shown in Ref. ${ }^{18}$

In what follows, we study the axisymmetric boundary layer over circular cones in hypersonic freestream flows. The freestream conditions and geometries are selected to match a subset of configurations used in the experiments conducted at $\mathrm{AFRL}^{2,36}$ and at AEDC. ${ }^{25}$ For this problem, the computational coordinates, $(\xi, \eta, \zeta)$, are defined as an orthogonal body-fitted coordinate system. The metric factors are defined as

$$
\begin{aligned}
& h_{1}=1+\kappa \eta, \\
& h_{3}=r_{b}+\eta \cos (\theta),
\end{aligned}
$$

where $\kappa$ denotes the streamwise curvature, $r_{b}$ is the local radius, and $\theta$ is the local half-angle along the axisymmetric surface, i.e., $\sin (\theta)=d r_{b} / d \xi$. For our study, the half angle, $\theta$, is $7^{\circ}$ and $\kappa=0$ in the frustum region. The end of the nose and beginning of the frustum is denoted as the juncture location that is defined as $\xi_{j}=R_{N} \pi / 2$. The streamwise coordinate within the nosetip region is represented by an angular coordinate defined as $\phi=\xi / R_{N}$. The nosetip Reynolds number, $R e_{R_{N}}=\bar{\rho}_{\infty} \bar{u}_{\infty} R_{N} / \mu_{\infty}$ is used to scale the energy gain. The length scale $L_{R_{N}}=R_{N} / \sqrt{R e_{R_{N}}}$ is used to normalize the spanwise disturbance wavelength defined as $\lambda=2 \pi r_{b} / m$.

\section{III.C. Transient Growth Results}

For a nonselfsimilar boundary layer such as the boundary layer over blunt cones, both the initial and final locations must be varied in order to obtain the overall picture of the optimal growth characteristics. ${ }^{23} \mathrm{~A}$ special feature of the transient growth analysis for the blunt cones of interest is that the results naturally split into two parts, one that deals with transient growth intervals that are limited to the nose region, where the results are expected to resemble those for the hemisphere forebody reported by Paredes et al., ${ }^{23}$ and a second one that deals with transient growth intervals that extend into the frustum region, where transition is observed in the experiments. Detailed transient growth results are first presented for the AFRL configurations in subsections III.C.1 and III.C.2 and finally, a brief summary of results is presented for the AEDC configurations in subsection III.C.3.

\section{III.C.1. Transient Growth Interval within the Nose Region}

Herein, transient growth results with initial and final disturbance locations within the nose region for the AFRL configurations introduced in Table 1 are investigated. The optimal mean total energy gain $\left(G_{E}^{\text {mean }}\right)$ and optimal mean kinetic energy gain $\left(G_{K}^{\text {mean }}\right)$ for the $R_{N}=15.24 \mathrm{~mm}$ and $R e_{\infty}=91.4 \times 10^{6} \mathrm{~m}^{-1}$ case are plotted in Figs. 8(a) and 8(b), respectively. Figure 8(a) shows that the highest total energy gain occurs for relatively short optimization intervals in the vicinity of the stagnation point, as indicated by the black line nearly parallel to the lower boundary of the plot. However, the kinetic energy budget for these perturbations initiated near the stagnation point is rather small. This fact is confirmed by the optimal mean kinetic energy gain $\left(G_{K}^{\text {mean }}\right)$ plot of Fig. 8(b). The optimal kinetic energy gain exhibits a maximum in the interior of the domain at $\phi_{0}=42.4^{\circ}$ that nearly coincides with the sonic location, $\phi_{M e=1}=41.4^{\circ}$. These results indicate the same features as the results reported by Paredes et al. ${ }^{23}$ for a hypersonic hemisphere forebody.

The optimal growth results for a specified inflow location $\xi_{0}$ of the flow are characterized in terms of the combination of azimuthal wavenumber $m$ and outflow location $\xi_{1}$ that lead to the maximum value of the energy gain. Thus, the effect of nosetip radius $R_{N}$ on the maximum value of the optimal energy gain, optimal wavenumber, and optimal growth interval, is analyzed next. Figure 9 shows the optimal total and kinetic energy gains as a function of the inflow location (Figs. 9(a) and 9(d)), as well as the corresponding azimuthal wavenumber (Figs. 9(c) and 9(e)) and the optimal growth interval (Figs. 9(c) and 9(f)). Results are shown for the $R_{N}=5.08 \mathrm{~mm}$ and $R_{N}=15.24 \mathrm{~mm}$ cones at the same freestream unit Reynolds number 


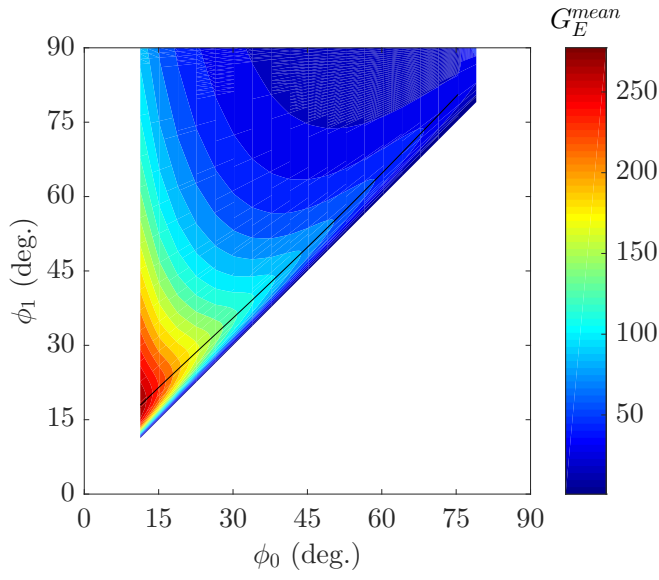

(a) Mean total energy gain.

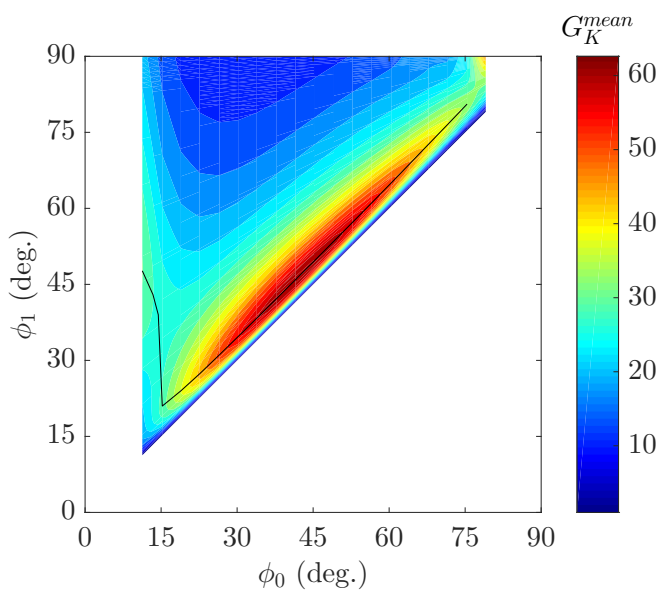

(b) Mean kinetic energy gain.

Figure 8. Contours of optimal mean total energy gain $G_{E}^{m e a n}$ and optimal mean kinetic energy gain $G_{K}^{m e a n}$ within the nose region of the $R_{N}=15.24 \mathrm{~mm}$ and $R e_{\infty}=91.4 \times 10^{6} \mathbf{m}^{-1}$ case. The solid line in the contour plot indicates the value of $\phi_{1}$ corresponding to maximum energy gain for a given $\phi_{0}$.

of $R e_{\infty}=91.4 \times 10^{6} \mathrm{~m}^{-1}$. Figure 9 (a) shows that both the total and kinetic energy gains are larger for the larger nose radius, although as shown in Fig. 9(d), the scaling is not perfectly linear because, as indicated by Paredes et al., ${ }^{24}$ small deviations from the linear scaling occur as a result of the differences in the ratio of boundary-layer thickness to the radius of the surface curvature. Figure 9(b) shows that the optimal azimuthal wavenumber is nearly twice as large for the larger nose radius cone in comparison with the case of the smaller nose radius. The scaling of the initial disturbance wavelength with $L_{R_{N}}$ shows a reasonable scaling with the boundary layer thickness. On the other side, the optimal growth interval plotted in Fig. 9(c) does not scale with $L_{R_{N}}$ (or $R_{N}$ ) as shown in Fig. $9(\mathrm{f})$, presumably because the ratio of boundary-layer thickness to the radius of the surface curvature plays an important role for this parameter.

\section{III.C.2. Transient Growth along the Frustum Region}

Next, transient growth across spatial intervals that extend into the frustum region is studied in detail for the AFRL configurations. In this case, we find it more convenient to plot the transient growth amplification in terms of the $N$-factor based on the total energy norm defined in Eq. (9). Figures 10(a) shows the $N$-factor contours for initial and final locations on the frustum for the $R_{N}=5.08 \mathrm{~mm}$ AFRL cone at $R e_{\infty}=91.4 \times 10^{6}$ $\mathrm{m}^{-1}$. Similar results for the larger nose radius, $R_{N}=15.24 \mathrm{~mm}$, are shown in Fig. 10 (b). The $N$-factor values are larger for the smaller nose radius cone except for initial and final locations near the juncture of the cone, i.e., beginning of the frustum. The $N_{E}$ values are larger than 5.5 in the studied range of parameters for the smaller nose radius case (Fig. 10(a)). For the larger nose radius case, the $N_{E}$ values are larger than 4.5 in the range of $\xi_{0}$ and $\xi_{1}$ values studied here, although the $N_{E}=3.5$ value is reached at smaller values of $\xi_{1}$ than for the smaller radius case when the initial disturbance location is near the juncture location $\xi_{0} \approx \xi_{j}$.

Figures 11(a) and 11(b) show a magnified view of the $N$-factor contours from Figs. 10(a) and 10(b), respectively, for initial and final locations in the vicinity of the juncture between the nose and the frustum of the cone. Both figures show different behavior of the transient growth amplification for disturbances initiated within the nose region $\left(\xi_{0}<\xi_{j}\right)$ and those that are initiated downstream of the juncture location $\left(\xi_{0} \geq \xi_{j}\right)$. The disturbances initiated in the nose have a maximum for very short optimal growth intervals, as previously shown in Fig. 9. For larger optimal growth intervals, the transient growth amplification first decreases and then increases again for $\xi_{1}>\xi_{j}$. On the other side, disturbances initiated downstream of the nose $\left(\xi_{0} \geq \xi_{j}\right)$ experience a monotonic increase in energy gain factor as $\xi_{1}$ is increased. Remarkably, disturbances initiated in the vicinity of the juncture location $\left(\xi_{0} \approx \xi_{j}\right)$ experience a quite rapid growth for the blunter case and short transient growth intervals (Fig. 11(b)), resulting in relatively significant values of $N_{E}$ just downstream of the juncture location.

The AFRL experiments investigated the effect of an azimuthally-periodic array of roughness elements mounted near the sonic point at $\phi=45^{\circ}$. To help gain some insight into the role of transient growth as a mechanism for roughness effects, we next examine the details of transient growth disturbances initiated 


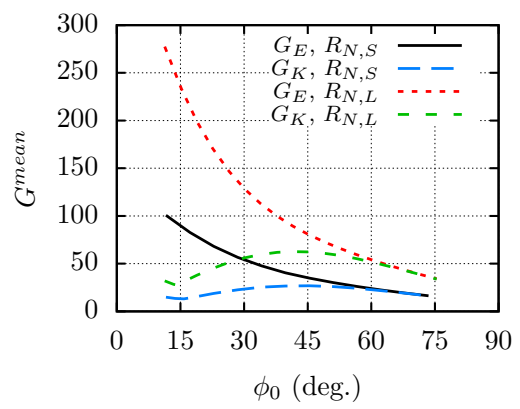

(a) Mean energy gain

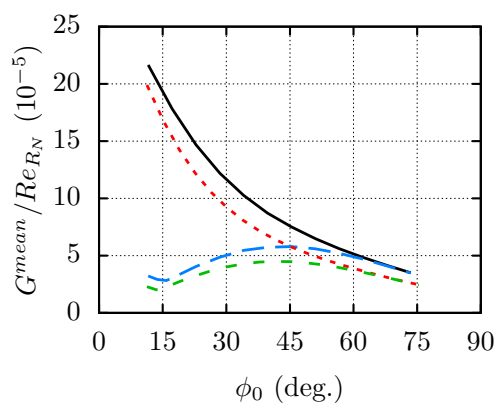

(d) Scaled mean energy gain

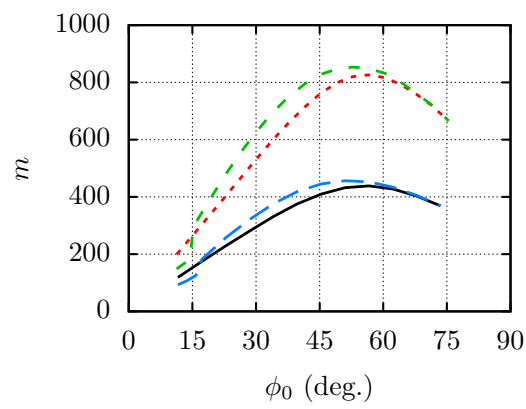

(b) Azimuthal wavenumber

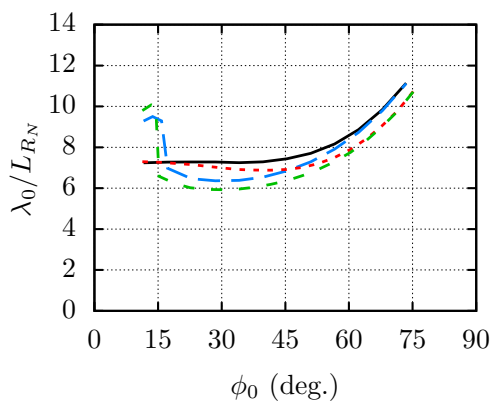

(e) Scaled initial wavelength

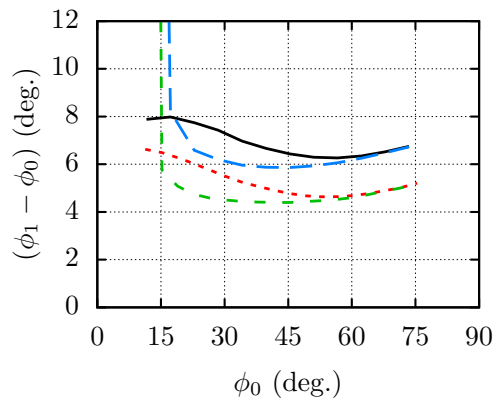

(c) Optimization interval

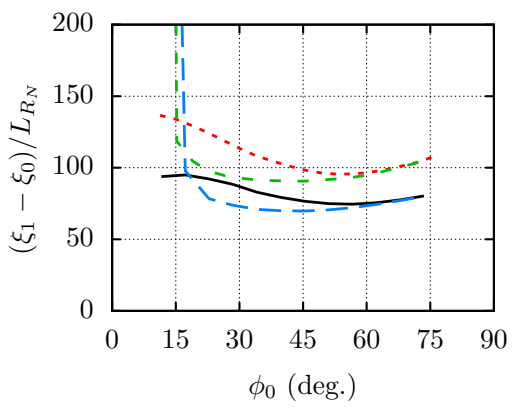

(f) Scaled optimization interval

Figure 9. Optimal mean energy gain and corresponding azimuthal wavenumber and optimization interval within the nose region for $R_{N, S}=5.08 \mathrm{~mm}$ and $R_{N, L}=15.24 \mathrm{~mm}$ cones at same freestream unit Reynolds number, $R e_{\infty}=91.4 \times 10^{6}$ $\mathbf{m}^{-1}$.

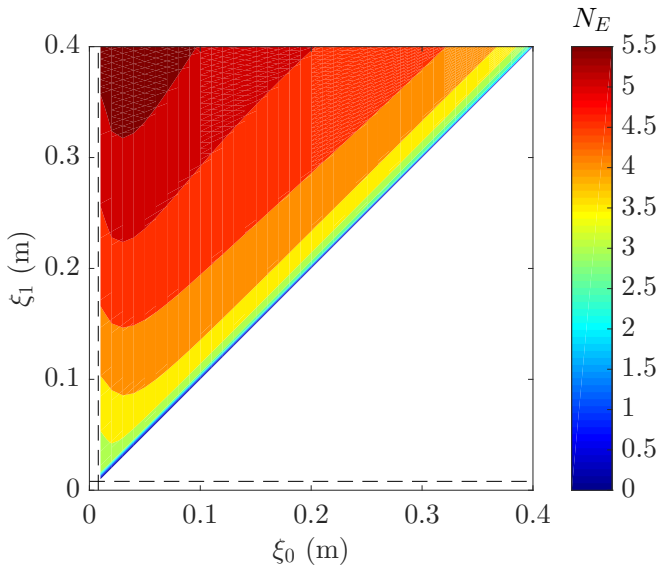

(a) $R_{N}=5.08 \mathrm{~mm}$

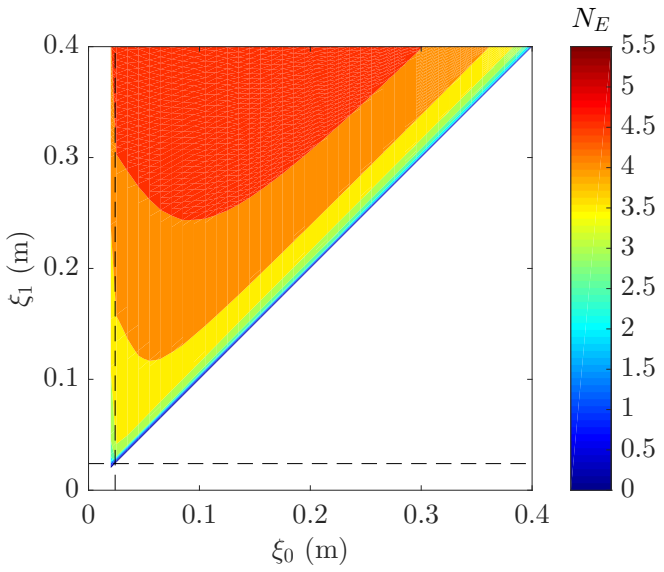

(b) $R_{N}=15.24 \mathrm{~mm}$

Figure 10. Contours of $N$-factor values defined as $N_{E}=1 / 2 \log \left(G_{E}^{o u t}\right)$ in the frustum region of the cone. The juncture location $\xi_{j}$ is marked with a vertical and a horizontal dashed line. The freestream unit Reynolds number is $R e_{\infty}=$ $91.4 \times 10^{6} \mathbf{m}^{-1}$. 


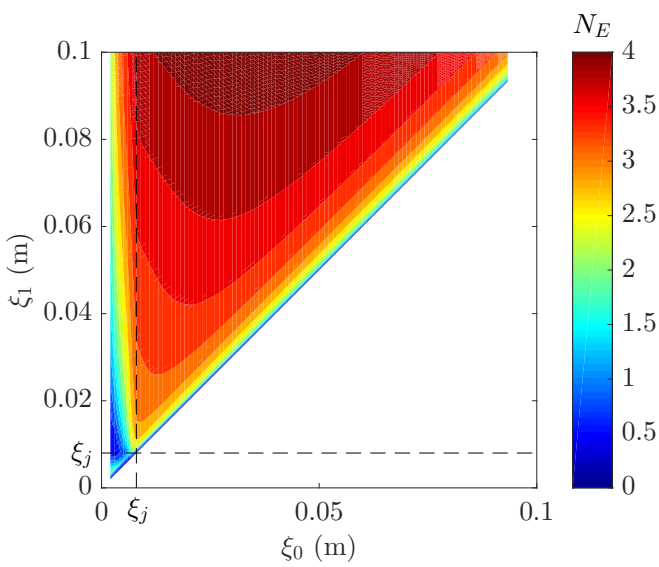

(a) $R_{N}=5.08 \mathrm{~mm}$.

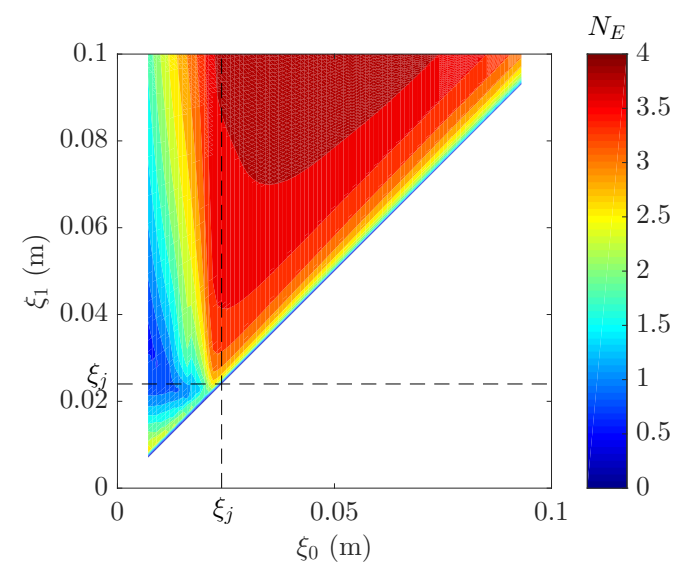

(b) $R_{N}=15.24 \mathrm{~mm}$.

Figure 11. Contours of $N$-factor values defined as $N_{E}=1 / 2 \log \left(G_{E}^{o u t}\right)$ in the vicinity of the nose region. The juncture location $\xi_{j}$ is marked with a vertical and a horizontal dashed line. The freestream unit Reynolds number is $R e_{\infty}=$ $91.4 \times 10^{6} \mathbf{m}^{-1}$.

in the nose region at $\phi_{0}=\xi_{0} / R_{N}=45^{\circ}$, which coincides with the location of the roughness array. A description of the experimental findings is deferred to section IV. Figure 12 shows the mean total energy gain and corresponding azimuthal wavenumber as a function of the optimal growth interval, $\Delta \xi=\xi_{1}-\xi_{0}$, for the four AFRL configurations shown in Table 1. Figure 12(a) shows that the trend previously observed for $\xi_{0}<\xi_{j}$ at $R e_{\infty}=91.4 \times 10^{6} \mathrm{~m}^{-1}$ (Fig. 11(b)) also applies at other Reynolds numbers. Specifically, the optimal energy gain has a maximum immediately downstream of the inflow station and then decays up to a plateau zone before increasing again for longer transient growth intervals. For the same freestream unit Reynolds number, the initial peak in optimal energy gain is larger for the larger nose radius cone than for the smaller nose radius case, but this situation is reversed for larger optimal growth intervals $(\Delta \xi>0.02$ $\mathrm{m})$. Figure 12(b) shows that the three regions translate into a discontinuous evolution of the corresponding azimuthal wavenumber. The scaling of the optimal mean total energy gain with $\operatorname{Re}_{R_{N}}$ (Fig. 12(c)) and of the corresponding initial azimuthal wavelength with $L_{R_{N}}$ (Fig. 12(d)) is reasonable for the cases with same nose radius $R_{N}=15.24 \mathrm{~mm}$ and different freestream unit Reynolds numbers, but not for the smaller nose radius case $\left(R_{N}=5.08 \mathrm{~mm}\right)$ and outside the nose region.

Figure 13 shows further details of the transient growth disturbances initiated in the nose at $\phi_{0}=45^{\circ}$ for the blunter cone at the highest $R e_{\infty}$ configuration $\left(R_{N}=15.24 \mathrm{~mm}\right.$ and $\left.R e_{\infty}=91.4 \times 10^{6} \mathrm{~m}^{-1}\right)$. Three cases based on the trends observed in Fig. 12 are plotted; namely, (A) $\Delta \xi=0.0030 \mathrm{~m}$ and $m=420$; (B) $\Delta \xi=0.023 \mathrm{~m}$ and $m=150$; and (C) $\Delta \xi=0.091 \mathrm{~m}$ and $m=80$. The case (A) corresponds to the first peak in mean energy gain that is larger for the larger nose radius cone (Fig. 12(a)). The evolution of the disturbance amplitude $\sqrt{E / E_{0}}$ for the case (A) shows a rapid rise to its peak value within a rather short distance from the initial disturbance location and a slower subsequent decay with values lower than 1 for streamwise locations in the frustum region. The initial optimal perturbation plotted in Fig. 13 is mostly contained within the boundary layer thickness; and hence, such initial disturbance profiles are better suited for excitation via surface roughness than some other cases where the initial profiles extend well outside of the boundary layer. The disturbance amplitude evolution of the case (B) has a smaller initial peak and then remains below 6 along the streamwise domain plotted in Fig. 13(a). The initial optimal perturbation associated with this case (B) is similar to that in case (A), but the peaks of the perturbation variables are located somewhat farther from the wall, which presumably makes this perturbation less likely to be excited via surface roughness. The energy gain evolution for case (C) $(\Delta \xi=0.091 \mathrm{~m}, m=80)$ shows rather small disturbance amplification near the inflow location and then a monotonic amplification up to $\xi=0.2 \mathrm{~m}$. Figure 13(b) shows that the optimal initial perturbation shape in case $(\mathrm{C})$ is more complex than that for cases (A) and (B), because the perturbation profiles for wall-normal and spanwise velocity components have two and three peaks instead of one and two peaks, respectively. Also, the perturbation profiles have a larger wall-normal extension and the peaks of these profiles are located farther from the wall. In summary, the results shown in Fig. 13 indicate that roughness-induced perturbations at $\phi_{0}=45^{\circ}$ can experience transient growth in a short interval within the nose region. The transient growth streaks can lead to the onset of 


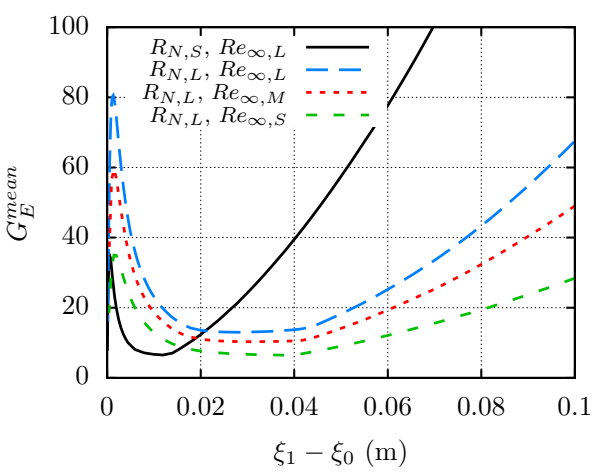

(a) Mean energy gain

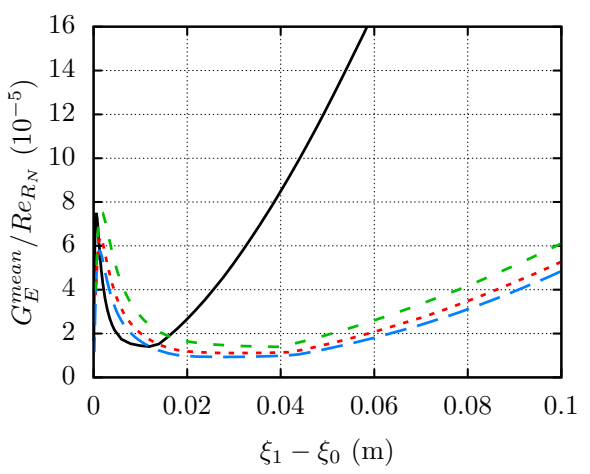

(c) Scaled mean energy gain

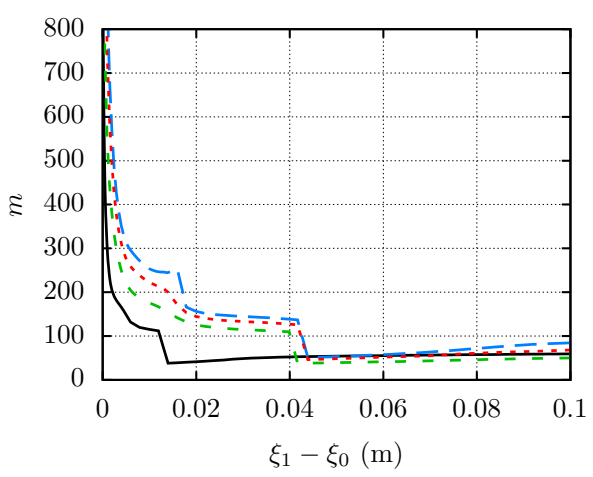

(b) Azimuthal wavenumber

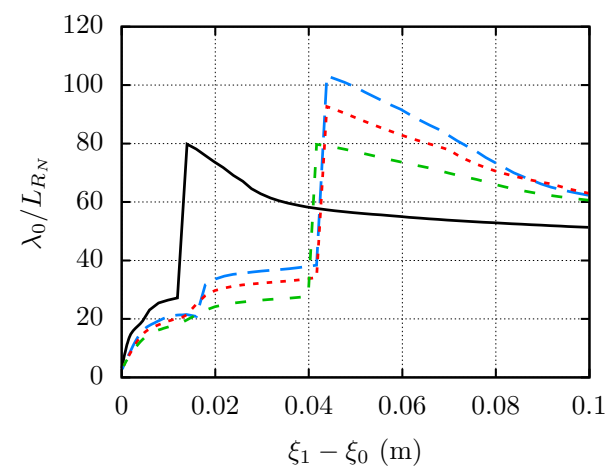

(d) Scaled initial wavelength

Figure 12. Optimal mean energy gain and corresponding azimuthal wavenumber with initial disturbance location $\theta_{0}=45^{\circ}\left(\xi_{0}=0.004 \mathrm{~m}\right.$ for the $R_{N}=5.08 \mathrm{~mm}$ cone and $\xi_{0}=0.012 \mathrm{~m}$ for the $R_{N}=15.24 \mathrm{~mm}$ cone). The legend refers to $R_{N, S}=5.08 \mathrm{~mm}, R_{N, L}=15.24 \mathbf{m m}, R e_{\infty, L}=91.4 \times 10^{6} \mathbf{m}^{-1}, R e_{\infty, M}=60.9 \times 10^{6} \mathbf{m}^{-1}, R e_{\infty, S}=30.5 \times 10^{6} \mathbf{m}^{-1}$. 
nonstationary streak instabilities that typically amplify rather rapidly and induce transition shortly after their onset; see Refs. 60-65 for details on secondary instability of streaks in high-speed boundary layers.

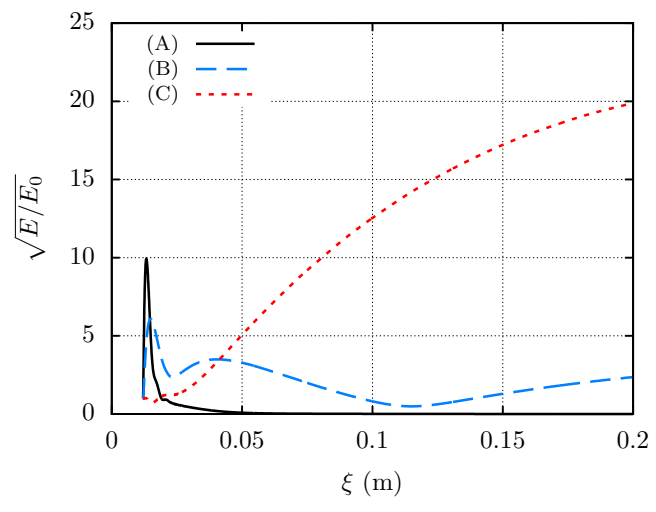

(a) Disturbance amplitude evolution
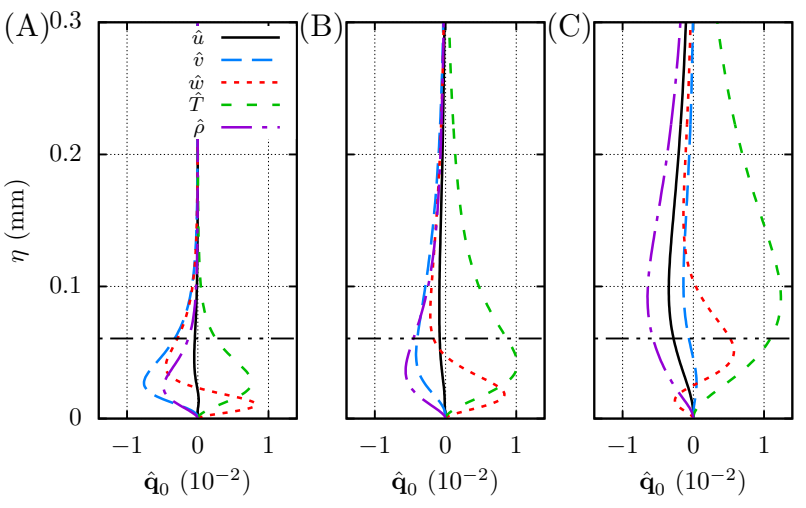

(b) Initial optimal perturbations

Figure 13. Evolution of the disturbance amplitude $\sqrt{E / E_{0}}$ and corresponding initial optimal perturbations for the $R_{N}=15.24 \mathrm{~mm}$ and $R e_{\infty}=91.4 \times 10^{6} \mathrm{~m}^{-1}$ configuration. The selected optimization intervals and azimuthal wavenumbers are (A) $\Delta \xi=0.0030 \mathrm{~m}$ and $m=420$; (B) $\Delta \xi=0.023 \mathrm{~m}$ and $m=150$; and (C) $\Delta \xi=0.091 \mathrm{~m}$ and $m=80$. The initial disturbance location is set at the nosetip at $\theta_{0}=45^{\circ}$ that corresponds to $\xi_{0}=0.012 \mathrm{~m}$. The horizontal, dash-dotted lines represent the boundary-layer edge, $\eta=\delta_{h}$.

Previously, disturbances initiated in the vicinity of the juncture location, $\xi_{j}=R_{N} \pi / 2$, were found to experience a larger growth for the larger nose radius cone and relatively short transient growth intervals. This finding is further investigated here. Figure 14 shows the mean gain in total energy and corresponding azimuthal wavenumber as a function of the optimal growth interval, $\Delta \xi=\xi_{1}-\xi_{0}$, for the four AFRL configurations shown in Table 1. Figure 14(a) shows in details that the trend observed in Fig. 11(b) is for $R e_{\infty}=91.4 \times 10^{6} \mathrm{~m}^{-1}$ is also found at other Reynolds numbers. Specifically, the energy gain has a monotonic increasing evolution as $\Delta \xi$ is increased, and the energy gain is larger for larger nose radius for relatively short optimization intervals $(\Delta \xi<0.13 \mathrm{~m})$. The difference between the energy gain values for the two nose radii $\left(R_{N}=5.08\right.$ and $\left.15.24 \mathrm{~mm}\right)$ and same freestream unit Reynolds number $\left(R e_{\infty}=91.4 \times 10^{6}\right.$ $\mathrm{m}^{-1}$ ) reaches about a factor of 2 for $\Delta \xi=0.05 \mathrm{~m}$. The azimuthal wavenumbers associated with the optimal energy gain values of Fig. 14(a) are shown in Fig. 14(b). The azimuthal wavenumber quickly decreases and as the length of the transient growth interval increases, albeit at a decreasing rate. Compared to the large bluntness cases $\left(R_{N}=15.24 \mathrm{~mm}\right)$, the smaller radius case $\left(R_{N}=5.08 \mathrm{~mm}, R e_{\infty}=91.4 \times 10^{6} \mathrm{~m}^{-1}\right)$ shows a notable, different behavior of both the optimal energy gain and the associated azimuthal wavenumbers. This difference is clearly observed in the scaled mean energy gain and scaled initial wavelength plotted in Figs. 14(c) and 14(d), respectively. The large nose radius cases $\left(R_{N}=15.24 \mathrm{~mm}\right)$ show a perfect scaling of both properties as the unit Reynolds number is varied, but the scaled values corresponding to the small radius case $\left(R_{N}=5.08 \mathrm{~mm}\right)$ are clearly different from the blunt nose cases. The reason for this poor scaling of the transient growth parameters with nose radius could be due to the lack of selfsimilarity of the boundary layer profiles near the nose.

Figure 15 shows further details of the transient growth disturbances initiated at the juncture location for the $R_{N}=15.24 \mathrm{~mm}$ and $R e_{\infty}=91.4 \times 10^{6} \mathrm{~m}^{-1}$ configuration. The three cases shown in Fig. 15 for the $R_{N}=15.24 \mathrm{~mm}$ case correspond to (A) $\Delta \xi=0.0295 \mathrm{~m}$ and $m=200$; (B) $\Delta \xi=0.0668 \mathrm{~m}$ and $m=150$; and (C) $\Delta \xi=0.2066 \mathrm{~m}$ and $m=110$. Figure 15(a) shows that the peak in disturbance amplitude moves slightly downstream as the optimal growth interval becomes longer from case (A) to case (C). This peak is barely reached at the end of the cone length for the case $(\mathrm{C})$. The optimal initial perturbations associated with the three cases are plotted in Fig. 15(b). The wall-normal extension and the peaks of these profiles are located closer to the wall for shorter optimal growth intervals, which makes them more closely related to roughness-induced perturbations. A wall-mounted device is not expected to generate a perturbation with the wall-normal extension of the optimal perturbation corresponding to case (C). Although not shown here, equivalent results for the small radius case $\left(R_{N}=5.08 \mathrm{~mm}, R e_{\infty}=91.4 \times 10^{6} \mathrm{~m}^{-1}\right)$ and an optimal growth interval of $\Delta \xi=0.0295 \mathrm{~m}$ show a peak disturbance amplitude equal to approximately one half of the peak value corresponding to the large radius cone $\left(R_{N}=15.24 \mathrm{~mm}, R e_{\infty}=91.4 \times 10^{6} \mathrm{~m}^{-1}\right)$ plotted in Fig. 15 . Similar to results for disturbances initiated near the sonic location, these results with $\xi_{0}=\xi_{j}$ indicate that 


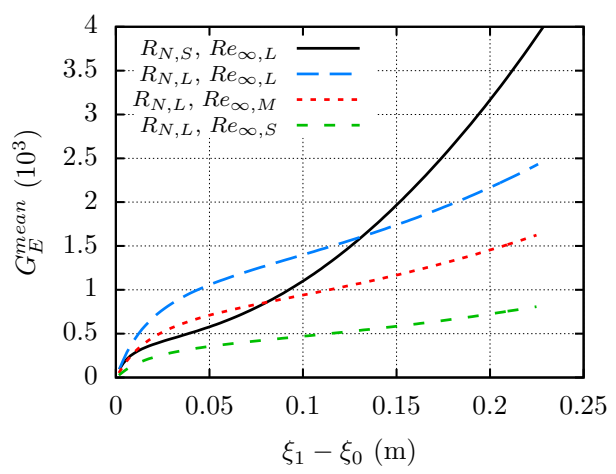

(a) Mean energy gain

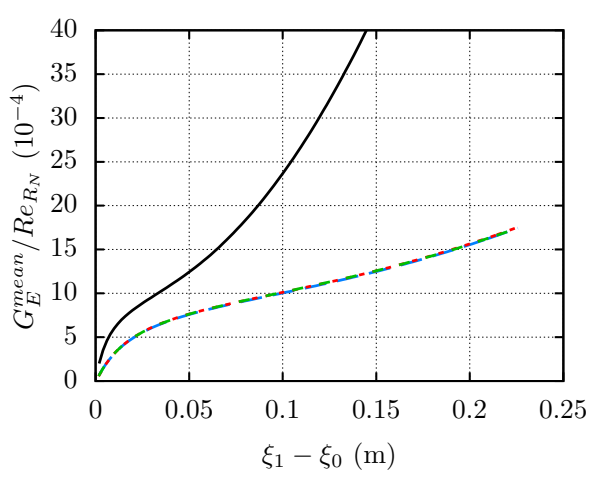

(c) Scaled mean energy gain

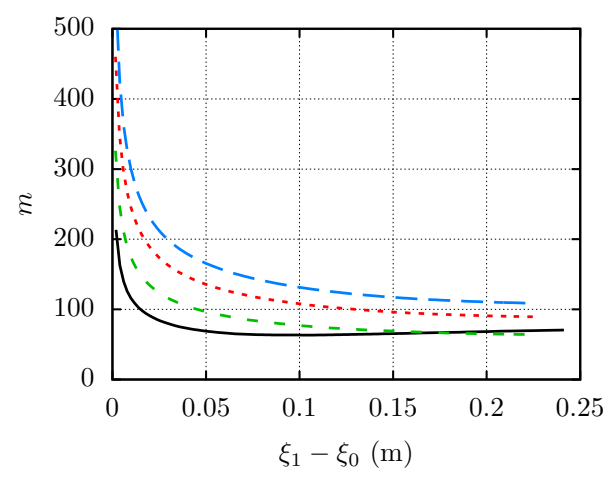

(b) Azimuthal wavenumber

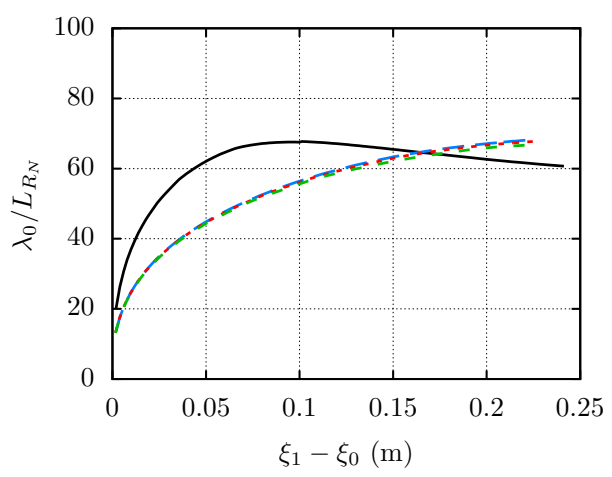

(d) Scaled initial wavelength

Figure 14. Optimal mean energy gain and corresponding azimuthal wavenumber with initial disturbance location set in the juncture at $\xi_{0}=\xi_{j}=R_{N} \pi / 2\left(\xi_{0}=0.008 \mathrm{~m}\right.$ for $R_{N}=5.08 \mathrm{~mm}$ and $\xi_{0}=0.024 \mathbf{m}$ for $R_{N}=15.24 \mathrm{~mm}$.) The legend refers to $R_{N, S}=5.08 \mathrm{~mm}, R_{N, L}=15.24 \mathbf{m m}, R e_{\infty, L}=9.14 \times 10^{7} \mathbf{m}^{-1}, R e_{\infty, M}=6.09 \times 10^{7} \mathbf{m}^{-1}, R e_{\infty, S}=3.05 \times 10^{7} \mathbf{m}^{-1}$. 
roughness-induced perturbations can experience greater transient growth for larger nosetip bluntness at the same freestream conditions. Therefore, transition onset could be driven by roughness-induced transient growth if the streak amplitude required for streak instabilities is reached.

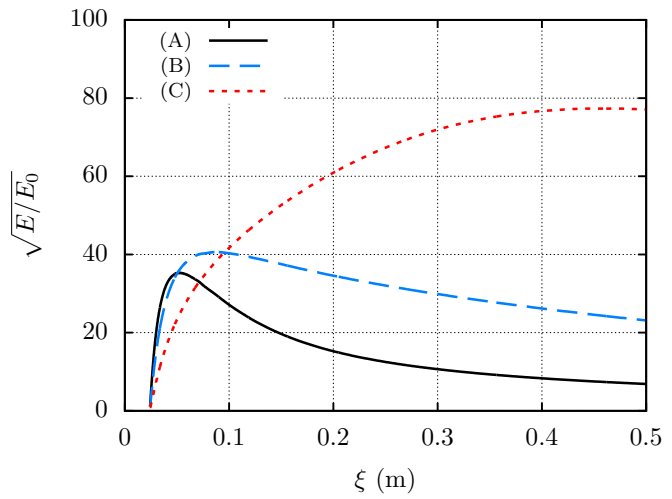

(a) Disturbance amplitude evolution
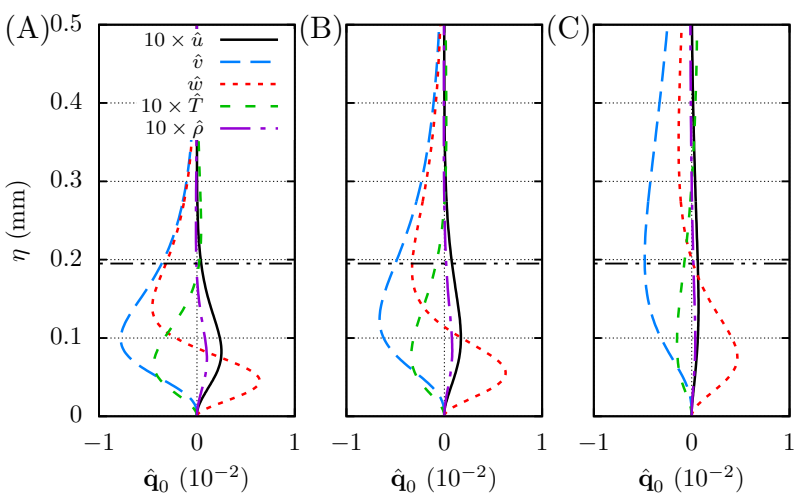

(b) Initial optimal perturbations

Figure 15. Evolution of the disturbance amplitude $\sqrt{E / E_{0}}$ and corresponding initial optimal perturbations for the $R_{N}=15.24 \mathrm{~mm}$ and $R e_{\infty}=9.14 \times 10^{7} \mathbf{m}^{-1}$ configuration. The selected optimization intervals and azimuthal wavenumbers are (A) $\Delta \xi=0.0295 \mathrm{~m}$ and $m=200$; (B) $\Delta \xi=0.0668 \mathrm{~m}$ and $m=150$; and (C) $\Delta \xi=0.2066 \mathrm{~m}$ and $m=110$. The initial disturbance location is set at the juncture $\xi_{0}=\xi_{j}$ that corresponds to $\xi_{0}=0.024 \mathrm{~m}$. The horizontal, dash-dotted lines represent the boundary-layer edge, $\eta=\delta_{h}$.

\section{III.C.3. Transient Growth on AEDC Configurations}

Transient growth results for the AFRL cones showed that disturbances initiated in the vicinity of the juncture between the nosetip and the frustum of the cone can experience a rather strong transient amplification in a short streamwise distance. This amplification is stronger for the larger nose radius case at same freestream conditions. Based on that observation, results for the AEDC configurations are focused on disturbances initiated at the juncture of the cones to study the trend with this different set of flow and geometry parameters. Figure 16 shows the evolution of optimal gain in mean energy gain as a function of the optimal growth interval for disturbances initiated at the juncture location. Again, as the nose radius of the cone is increased, the optimal energy gain becomes notably larger. Figure 16(b) indicates that the scaled energy gain values, $G_{E}^{m e a n} / R e_{R_{N}}$, are smaller for larger nose radius cases, indicating a nonlinear increase in optimal energy gain with increasing nose bluntness. This trend is similar to the trend in Fig. 14(c) for the AFRL configurations.

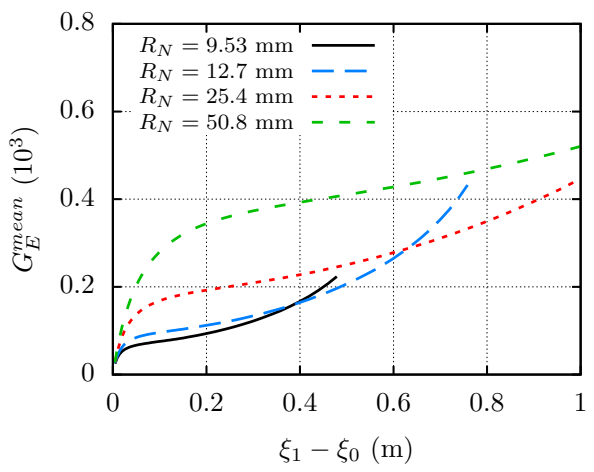

(a) Mean energy gain

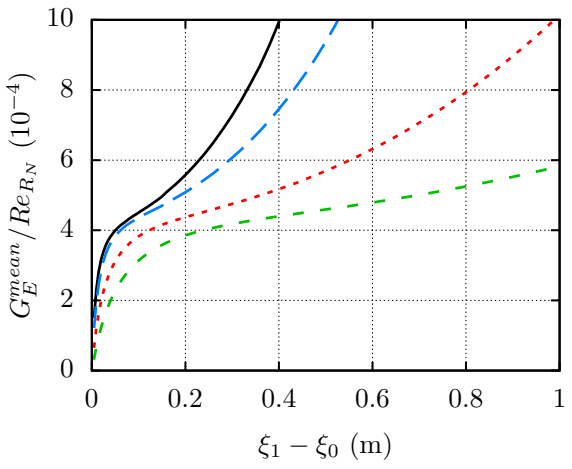

(b) Scaled mean energy gain

Figure 16. Optimal mean energy gain with initial disturbance location set at the juncture $\xi_{0}=\xi_{j}=R_{N} \pi / 2$ for the AEDC configurations $\left(\xi_{0}=0.015 \mathbf{m}\right.$ for $R_{N}=9.53 \mathbf{m m}, \xi_{0}=0.020 \mathbf{m}$ for $R_{N}=12.7 \mathbf{m m}, \xi_{0}=0.040 \mathbf{m}$ for $R_{N}=25.4 \mathbf{m m}$, and $\xi_{0}=0.080 \mathbf{m}$ for $\left.R_{N}=50.8 \mathbf{m m}\right)$. The freestream unit Reynolds number is $R e_{\infty} \approx 17.5 \times 10^{6} \mathbf{m}^{-1}$. 


\section{Roughness Effects on Laminar-Turbulent Transition on Blunt Cones}

Between 1978 and 1982, K. F. Stetson performed a total of 196 sharp- and blunt-cone experiments on a thin-walled $8^{\circ}$ half-angle, $4 \mathrm{in}$. $(0.1016 \mathrm{~m})$ diameter base cone at zero angle of attack in the AFRL Mach 6 High Reynolds Number facility. These measurements were initially reported in a 1983 paper $^{2}$ and recently Jewell \& $\mathrm{Kimmel}^{36}$ performed a computational analysis indicating that neither the Mack second mode nor the first mode were likely to be the dominant instability mechanism for nosetips with radius larger than $1 \mathrm{~mm}$, for which transition was nevertheless consistently observed once sufficient freestream Reynolds numbers were reached. Alternate physical mechanisms may explain these results, and include transient growth or mean flow distortion from roughness.

More recently, experiments have been performed in the same facility on a $7^{\circ}$ half-angle variable-bluntness cone at zero angle of attack with similar nosetip bluntness values, which have also been shown to transition from laminar to turbulent flow without significant predicted amplification of first- or second-mode instability for smooth nosetips with radius larger than $1 \mathrm{~mm}$ and sufficient freestream Reynolds numbers. ${ }^{66} \mathrm{~A}$ subset of the smooth cases, with $R_{N}=5.08 \mathrm{~mm}$ and $R_{N}=15.24 \mathrm{~mm}$, have been repeated with $15 \mu \mathrm{m}$ laser-machined roughness elements of approximately cubical shape located on the circle at 45 degrees from the apex of the spherical tip, which is near the sonic line for zero angle of attack. The array of roughness elements has been designed following the transient growth results of subsection III.C. The number of roughness elements has been selected to match the disturbance azimuthal wavenumber that lead to significant transient growth at the same conditions. Specifically, 210 roughness elements were mounted on the small radius cone $\left(R_{N}=5.08\right.$ $\mathrm{mm})$ and 420 roughness elements on the large radius cone $\left(R_{N}=15.24 \mathrm{~mm}\right)$. A picture of the small radius nosetip with the array of roughness elements is shown in Fig. 17.

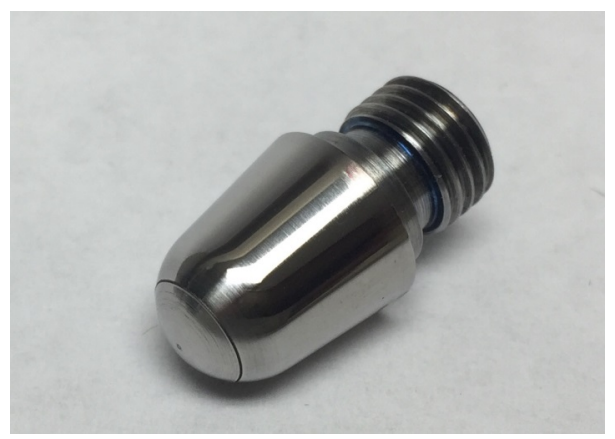

Figure 17. Nosetip of $R_{N}=5.08 \mathrm{~mm}$ with 210 equally spaced $15 \mu \mathrm{m}$ cubical roughness elements that were laser-machined circumferentially at $45^{\circ}$ from the apex of the spherical tip.

The presence of roughness elements on the $R_{N}=5.08 \mathrm{~mm}$ cone had little discernible effect on the experimentally observed transition location or heating levels across the operating conditions of the Mach 6 Hi Re Facility. In contrast, roughness elements of the same shape and dimension on the $R_{N}=15.24 \mathrm{~mm}$ cone had a profound effect on transition above a critical Reynolds number. This effect is illustrated in Figs. 18(a) and 18(b), which present surface heat flux distributions measured by Medtherm coaxial thermocouples from pairs of rough and smooth experiments, with roughness-height $R e_{k k}$ values matched to within $3 \%$ between the two sets. In Fig. 18(a), the rough $\left(k / \delta_{h}=0.381, R e_{k k}=294\right)$ and smooth surface heat transfer for the $R_{N}=5.08 \mathrm{~mm}$ cone overlap. In Fig. 18(b), the rough $\left(k / \delta_{h}=0.225, R e_{k k}=287\right)$ and smooth case for the $R_{N}=15.24 \mathrm{~mm}$ cone are quite different, with the rough tip resulting in nearly immediate transition onset, while the equivalent smooth tip results in laminar flow nearly to the end of the cone. This result indicates that the onset of transition in this data is associated with the combined effects of bluntness and roughness.

\section{Summary and Concluding Remarks}

A collaborative effort by the NATO STO group AVT-240 on Hypersonic Boundary-Layer Transition Prediction was devoted to bluntness effects on transition over circular cones at zero angle of attack. This paper presented an overview of that work, which consisted of transition measurements on blunt cones models with varying degrees of nosetip bluntness, measurements pertaining to the combined effects of nose bluntness and controlled surface roughness on the nose tip, and theoretical analysis of optimal transient growth on blunt cones. 


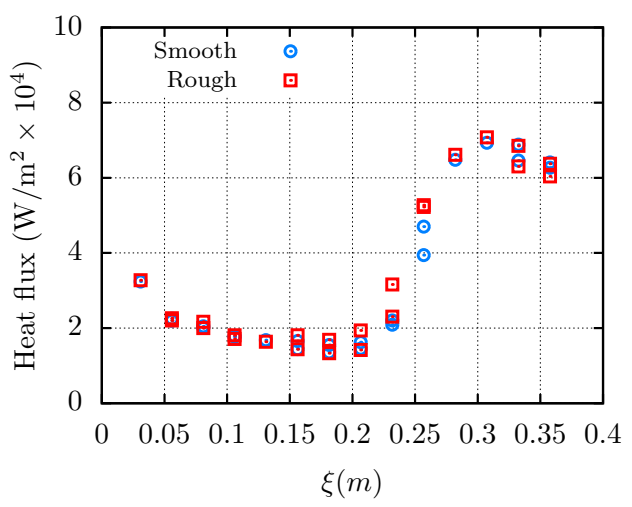

(a) $R_{N}=5.08 \mathrm{~mm}$. Smooth case with $R e_{\infty}=$ $66.0 \times 10^{6} \mathrm{~m}^{-1}$ and rough case with 210 roughness elements and $R e_{\infty}=66.5 \times 10^{6} \mathrm{~m}^{-1}$ that results in $R e_{k k}=294$.

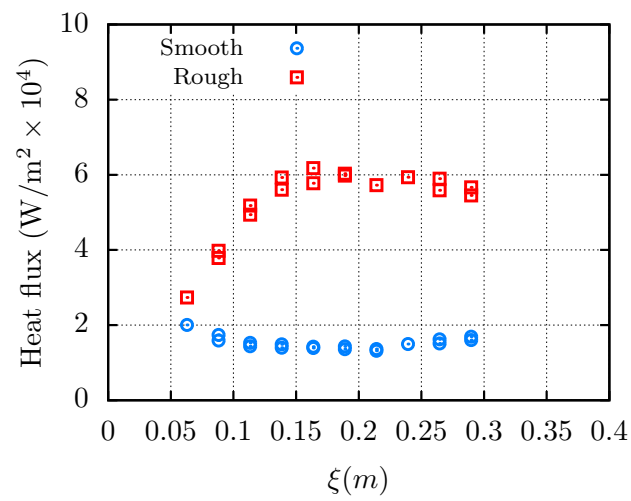

(b) $R_{N}=15.24 \mathrm{~mm}$. Smooth case with $R e_{\infty}=$ $76.9 \times 10^{6} \mathrm{~m}^{-1}$ and rough case with 420 roughness elements and $R e_{\infty}=75.8 \times 10^{6} \mathrm{~m}^{-1}$ that results in $R e_{k k}=287$.

Figure 18. Heat flux distributions for a $7^{\circ}$ half-angle cone at $M_{\infty}=5.9$ with nosetip of (a) $R_{N}=5.08 \mathrm{~mm}$ and (b) $R_{N}=15.24 \mathrm{~mm}$ for a smooth tip and a tip with a row of equally spaced $15 \mu \mathrm{m}$ cubical roughness elements.

The following observations can be made on the basis of wind tunnel measurements at freestream Mach numbers of 6,9 , and 10 :

(i) The recent measurements are consistent with the known trend of an initially downstream movement in the transition onset location as the nosetip bluntness is increased. This trend confirms the stabilizing effect of nose bluntness on the amplification of Mack mode, i.e., second-mode instability waves.

(ii) The measurements also confirm the well-known observation concerning a reversal in the above trend at sufficiently large value of the nosetip Reynolds number and the transition reversal phenomenon cannot be explained via the hydrodynamic stability paradigm, due to the absence of an adequately significant modal amplification of first-mode, second-mode, and entropy-layer instabilities.

(iii) Experimental measurements indicate that, in the transition reversal regime, boundary layer transition over the frustum of the cone is highly sensitive to wall roughness. The onset of transition appears to be dominated by disturbances that originate in the vicinity of the nosetip, suggesting roughness-induced transient growth as a likely cause for laminar-turbulent transition.

Optimal transient growth analysis has been conducted for the laminar flow based on the solution of the Navier-Stokes equations over a $7^{\circ}$ half-angle variable-bluntness cone at zero angle of attack with bluntness and freestream conditions corresponding to a subset of the experiments of Stetson ${ }^{2}$ at the AFRL Mach 6 High Reynolds Number facility and $R e_{\infty}=30.5-91.4 \times 10^{6} \mathrm{~m}^{-1}$ and of Marineau et al. ${ }^{25}$ at the AEDC Tunnel 9 facility with the Mach 10 nozzle and $R e_{\infty} \approx 17.5 \times 10^{6} \mathrm{~m}^{-1}$. Estimates of maximum possible transient growth are obtained through a dual optimization ${ }^{23,24}$ that varies both the initial and final locations of the transient growth interval. The following conclusions may be drawn on the basis of the transient growth analysis:

(i) The optimal growth results can be naturally divided into two parts. The first part deals with transient growth intervals limited to the nose region, and second part applies to optimal growth intervals that extend to the frustum region that encompasses the range of measured transition locations.

(ii) Results for optimal growth intervals within the nose region are similar to the findings by Paredes et al. ${ }^{23}$ for a hemispherical forebody. Specifically, the highest predicted growth in total energy corresponds to relatively short transient growth intervals in the vicinity of the stagnation point, but the kinetic energy growth associated with these perturbations is rather small. The maximum growth in disturbance kinetic energy is found for perturbations initiated near the sonic point at an angle of $42.4^{\circ}$ from the apex of the spherical tip. Furthermore, the wall-normal profiles of optimal initial perturbations associated with the maximum energy gain are concentrated within the boundary layer thickness, i.e., close to the wall. More important, at a fixed set of freestream conditions, increased nose bluntness results in higher transient amplification within the nose region. 
(iii) Based on the transient growth analysis for both AFRL and AEDC configurations, one may conclude that disturbances that are initiated in the vicinity of the juncture between the nosetip and the frustum also exhibit a greater transient amplification for larger values of nosetip bluntness, but only for relatively short optimization intervals. Furthermore, for the large nosetip bluntness, the wall-normal extension of the optimal initial perturbations is small, indicating that wall roughness may be able to induce such perturbations.

We note that the actual generation (i.e., receptivity) of the transient growth disturbances has not been addressed in this paper. Disturbance profiles resulting from realistic external disturbances usually result in a suboptimal transient growth. In that regard, the present results based on optimal initial disturbances may be viewed as providing an upper bound on the nonmodal amplification due to spanwise periodic disturbances.

Finally, the roughness effects on transition reversal over a $7^{\circ}$ half-angle blunt cone at hypersonic flow is experimentally investigated in the AFRL Mach 6 High Reynolds Number facility. Transition measurements were made with an azimuthally periodic array of $15 \mu \mathrm{m}$, laser-machined roughness elements of approximately cubical shape located at $45^{\circ}$ from the apex of the spherical tip, which nearly coincides with the sonic location. The roughness array spacing ( $m=210$ for $R_{N}=5.08 \mathrm{~mm}$ and $m=420$ for $R_{N}=15.24 \mathrm{~mm}$ ) was designed to match the disturbance azimuthal wavenumber that is predicted to induce large transient growth under the respective conditions. The following observations can be made on the basis of these measurements:

(i) The roughness array on the small nosetip bluntness cone showed no effect on the transition location at $R e_{\infty} \approx 66 \times 10^{6} \mathrm{~m}^{-1}$ (i.e., $k / \delta_{h}=0.381, R e_{k k}=294$ ).

(ii) On the other hand, the experiments conducted with the large nosetip bluntness with $R e_{\infty} \approx 76 \times 10^{6}$ $\mathrm{m}^{-1}$ (i.e., $k / \delta_{h}=0.225, R e_{k k}=287$ ) showed a prominent effect of the roughness elements on the transition onset, moving the transition front close to the nosetip, whereas the smooth tip baseline for the same blunter cone has resulted in a laminar flow over the majority of the cone.

(iii) The above findings indicate that the transition onset is associated with the combined effects of bluntness and roughness.

Finally, we note that wakes behind sufficiently large discrete roughness elements can sustain large modal amplification of streak instabilities as shown by Choudhari et al. ${ }^{60,62}$ but the level of amplification is sensitive to the roughness parameters and other flow details. ${ }^{61}$ Because the measured onset of transition during the AFRL experiments was within a short distance behind the roughness array, wake instability may have played a role in the large upstream shift in transition location; and we are currently investigating that possibility via more detailed computations. However, a broader set of measurements involving controlled surface roughness would be useful in determining whether or not naturally occurring micro-roughness over the model surface may have been responsible for the observed transition reversal in other blunt cone experiments. Effects of roughness location in the vicinity of the nosetip and the juncture location would be an important aspect of such a parametric study. Flow visualizations accompanied by dynamic measurements, i.e., high frequency response heat transfer or pressure measurements, in the region between the controlled surface roughness and the onset of transition would go a long way in providing valuable clues regarding the physical mechanisms at play. Finally, performing parallel measurements in both quiet and conventional wind tunnel facilities would provide further information regarding the role of freestream disturbances in the transition reversal phenomenon.

\section{Acknowledgments}

Part of this research was sponsored by the NASA Transformational Tools and Technologies (TTT) Project of the Transformative Aeronautics Concepts Program (TACP) of the Aeronautics Research Mission Directorate (ARMD). A portion of the computational resources supporting this work were provided by the NASA High-End Computing (HEC) Program through the NASA Advanced Supercomputing (NAS) Division at Ames Research Center. The authors would like to thank Test Resource Management Center (TRMC) Test and Evaluation/Science and Technology (T\&E/S\&T) Program for their support. E. C. Marineau's work was funded by T\&E/S\&T Program through the High Speed Systems Test (HSST) area as part of the Center of Testing Excellence (CoTE). We also acknowledge Benxin Wu and his group at Purdue University for lasermachining the nose tip roughness elements. J. S. Jewell was supported by the NRC Research Associateship for part of this work. 


\section{References}

${ }^{1}$ Schneider, S., "Hypersonic laminar-turbulent transition on circular cones and scramjet forebodies," Prog. Aero. Sciences, Vol. 40, 2004, pp. 1-50.

${ }^{2}$ Stetson, K., "Nosetip bluntness effects on cone frustum boundary layer transition in hypersonic flow," AIAA Paper 83-1763, 1983.

${ }^{3}$ Morkovin, M., Reshotko, E., and Herbert, T., "Transition in open flow systems - A reassessment," Bull. Am. Phys. Soc., Vol. 39, 1994, pp. 1882.

${ }^{4}$ Reshotko, E., "Boundary layer transition, instability and control," AIAA Paper 94-0001, 1994.

${ }^{5}$ Reshotko, E. and Tumin, A., "Spatial theory of optimal disturbances in a circular pipe flow," Phys. Fluids, Vol. 13, 2001, pp. 991-996.

${ }^{6}$ Avila, K., Moxey, D., De Lozar, A., Avila, M., Barkely, D., and Hof, B., "The onset of turbulence in pipe flow," Science, Vol. 333, 2011, pp. 192-196.

${ }^{7}$ Reshotko, E., "Preliminary experimental study of disturbances in a laminar boundary layer due to distributed surface roughness," AIAA Paper 81-1224, 1981.

${ }^{8}$ White, E., "Transient growth of stationary disturbances in a flat plate boundary layer," Phys. Fluids, Vol. 14, No. 12, 2002, pp. 4429-4439. 2014.

${ }^{9}$ Sharp, N. and White, E., "Roughness-induced transient growth on a hypersonic blunt cone," AIAA Paper 2014-0432,

${ }^{10}$ Murphy, J. and Rubesin, M., "Re-evaluation of heat-transfer data obtained in flight test of heat-sink shielded re-entry vehicles," J. Spacecraft, Vol. 3, No. 1, 1966, pp. 53-60.

${ }^{11}$ Reshotko, E. and Tumin, A., "The blunt body paradox - A case for transient growth," Proc. of the IUTAM LaminarTurbulent Symposium V, edited by H. Fasel and W. Saric, Sedona, AZ, USA, 2000, pp. 403-408.

${ }^{12}$ Schneider, S., "Hypersonic boundary-layer transition on blunt bodies with roughness," AIAA Paper 2008-0501, 2008.

${ }^{13}$ Hollis, B., "Blunt-body entry vehicle aerothermodynamics: Transition and turbulent heating," J. Spacecraft Rockets, Vol. 49, No. 3, 2012, pp. 435-449.

${ }^{14}$ Ellingsen, T. and Palm, E., "Stability of linear flow," Phys. Fluids, Vol. 18, No. 4, 1975, pp. 143-148.

${ }^{15}$ Stuart, J., "The production on intense shear layers by vortex stretching and convection," AGARD Report No. 514 (also: National Phys. Lab. Aeronaut. Res. Rep. 1147), 1965.

${ }^{16}$ Landahl, M., "A note on algebraic instability of inviscid parallel shear flows," J. Fluid Mech., Vol. 98, 1980, pp. $243-251$.

${ }^{17}$ Tumin, A. and Reshotko, E., "Optimal disturbances in compressible boundary layers," AIAA J., Vol. 41, 2003, pp. 23572363.

${ }^{18}$ Paredes, P., Choudhari, M., Li, F., and Chang, C.-L., "Optimal growth in hypersonic boundary layers," AIAA J., Vol. 54, No. 10, 2016, pp. 3050-3061.

${ }^{19}$ Schmid, P. and Henningson, D. S., Stability and Transition in Shear Flows, Springer, New York, 2001.

${ }^{20}$ Schmid, P. J., "Nonmodal stability theory," Ann. Rev. Fluid Mech., Vol. 39, 2007, pp. 129-162.

${ }^{21}$ Reshotko, E. and Tumin, A., "Role of transient growth in roughness-induced transition," AIAA J., Vol. 42, 2004, pp. 766770.

${ }^{22}$ Andersson, P., Berggren, M., and Henningson, D., "Optimal disturbances and bypass transition in boundary layers," Phys. Fluids, Vol. 11, 1999, pp. 134-150.

${ }^{23}$ Paredes, P., Choudhari, M., and Li, F., "Blunt-body paradox and transient growth on a hypersonic spherical forebody," Phys. Rev. Fluids, Vol. 2, 2017, pp. 053903-1-24.

${ }^{24}$ Paredes, P., Choudhari, M., and Li, F., "Blunt-body paradox and improved application of transient growth framework," AIAA J., Vol. (in review), 2017.

${ }^{25}$ Marineau, E., Moraru, C., Lewis, D., Norris, J., Lafferty, J., Wagnild, R., and Smith, J., "Mach 10 boundary-layer transition experiments on sharp and blunted cones," AIAA Paper 2014-3108, 2014.

${ }^{26}$ Aleksandrova, E., Novikova, A., Utyzhnikov, S., and Fedorov, A., "Experimental study of the laminar turbulent transition on a blunt cone," J. Appl. Mech. Tech. Phys., Vol. 55, No. 3, 2014, pp. 375-385.

${ }^{27}$ Softley, E., Graber, B., and Zempel, R., "Experimental observation of transition of the hypersonic boundary layer," AIAA J., Vol. 7, No. 2, 1969, pp. 254-263.

${ }^{28}$ Softley, E., "Boundary layer transition on hypersonic blunt, slender cones," AIAA Paper 69-0705, 1969.

${ }^{29}$ Stetson, K., "Hypersonic boundary layer transition experiments," AFWAL-TR-80-3062, 1980.

${ }^{30}$ Muir, J. and Trujillo, A., "Experimental investigation of the effects of nose bluntness, free-stream unit Reynolds number, and angle of attack on cone boundary layer transition at a Mach number of 6," AIAA Paper 72-0216, 1972.

${ }^{31}$ Stetson, K. and Rushton, G., "Shock tunnel investigation of boundary layer transition at M=5.5," AIAA J., Vol. 5, 1967, pp. 899-906.

${ }^{32}$ Rotta, N., "Effects of nose bluntness on the boundary layer characteristics of conical bodies at hypersonic speeds," NYU-AA-66-66, 1966.

${ }^{33}$ Malik, M., Spall, R., and Chang, C.-L., "Effect of nose bluntness on boundary layer stability and transition," AIAA Paper 90-0112, 1990.

${ }^{34}$ Herbert, T. and Esfahanian, V., "Stability of hypersonic flow over a blunt body," Tech. rep., AGARD CP-514. Paper 28. Theoretical and experimental methods in hypersonic flows., 1994.

${ }^{35}$ Marineau, E., "Prediction methodology for second-mode-dominated boundary-layer transition in hypersonic wind tunnels," AIAA J., Vol. 55, No. 2, 2017, pp. 484-499.

${ }^{36}$ Jewell, J. and Kimmel, R., "Boundary layer stability analysis for Stetson's Mach 6 blunt cone experiments," J. Spacecraft Rockets, Vol. 54, No. 1, 2017, pp. 258-265. 

2005.

${ }^{37}$ Johnson, H. and Gandler, G., "Hypersonic boundary layer stability analysis using PSE-Chem," AIAA Paper 2005-5023,

${ }^{38}$ Balakumar, P. and Chou, A., "Transition prediction in hypersonic boundary layers using receptivity and freestream spectra," AIAA J., Vol. (ahead of print), 2017, pp. 1-16.

${ }^{39}$ Zanchetta, M., Kinetic heating and transition studies and hypersonic speeds, Ph.D. thesis, Imperial College of Science, Technology and Medicine, London, 1996.

${ }^{40}$ Grossir, G., Pinna, F., Bonucci, G., Regert, T., Rambaut, P., and Chazot, O., "Hypersonic boundary layer transition on a 7 degree half-angle cone at Mach 10," AIAA Paper 2014-2774, 2014.

${ }^{41}$ Dietz, G. and Hein, S., "Entropy-layer instabilities over a blunted flat plate in supersonic flow," Phys. Fluids, Vol. 11, No. 1, 1999, pp. 7-9.

${ }^{42}$ Fedorov, A. and Tumin, A., "Evolution of disturbances in entropy layer on blunted plate in supersonic flow," AIAA J., Vol. 42, No. 1, 2004, pp. 89-94.

${ }^{43}$ Wright, M., Candler, G., and Bose, D., "Data-parallel line relaxation method for the Navier-Stokes equations," AIAA J., Vol. 36, No. 9, 1998, pp. 1603-1609.

${ }^{44}$ Johnson, H., Thermochemical interactions in hypersonic boundary layer stability, Ph.D. thesis, Univ. of Minnesota, 2000.

${ }^{45}$ Johnson, H., Seipp, T., and Candler, G., "Numerical study of hypersonic reactig boundary layer transition on cones," Phys. Fluids, Vol. 10, No. 10, 1998, pp. 2676-2685.

${ }^{46}$ Pralits, J., Airiau, C., Hanifi, A., and Henningson, D., "Sensitivity analysis using adjoint parabolized stability equations for compressible flows," Flow Turbul. Combust., Vol. 65, 2000, pp. 183-210.

${ }^{47}$ Tempelmann, D., Hanifi, A., and Henningson, D., "Spatial optimal growth in three-dimensoinal boundary layers," $J$. Fluid Mech., Vol. 646, 2010, pp. 5-37.

${ }^{48}$ Tempelmann, D., Hanifi, A., and Henningson, D., "Spatial optimal growth in three-dimensoinal compressible boundary layers," J. Fluid Mech., Vol. 704, 2012, pp. 251-279.

${ }^{49}$ Luchini, P., "Reynolds-number-independent instability of the boundary layer over a flat surface: Optimal perturbations," J. Fluid Mech., Vol. 404, 2000, pp. 289-309.

${ }^{50} \mathrm{Li}$, F. and Malik, M., "Mathematical nature of parabolized stability equations," R. Kobayashi (Ed.), Laminar-Turbulent Transition, Springer, 1994, pp. 205-212.

${ }^{51}$ Li, F. and Malik, M., "On the nature of the PSE Approximation," Theor. Comp. Fluid Dyn., Vol. 8, 1996, pp. $253-273$.

${ }^{52} \mathrm{Li}, \mathrm{F}$. and Malik, M., "Spectral analysis of parabolized stability equations," Compt. Fluids, Vol. 26 (3), 1997, pp. $279-297$. 53.

${ }^{53}$ Haj-Hariri, H., "Characteristics analysis of the parabolized stability equations," Stud. Appl. Math., Vol. 92, 1994, pp. 41-

${ }^{54}$ Bagheri, S. and Hanifi, A., "The stabilizing effect of streaks on Tollmien-Schlichting and oblique waves: A parametric study," Phys. Fluids, Vol. 19, 2007, pp. 078103-1-4.

${ }^{55}$ Zuccher, S., Tumin, A., and Reshotko, E., "Parabolic approach to optimal perturbations in compressible boundary layers," J. Fluid Mech., Vol. 556, 2006, pp. 189-216.

${ }^{56}$ Mack, L. M., "Boundary Layer Stability Theory," Tech. Rep. 900-277, Jet Propulsion Lab., Pasadena, CA, 1969.

${ }^{57}$ Hanifi, A., Schmid, P., and Henningson, D., "Transient growth in compressible boundary layer flow," Phys. Fluids, Vol. 8, 1996, pp. 51-65.

${ }^{58}$ Hermanns, M. and Hernández, J. A., "Stable high-order finite-difference methods based on non-uniform grid point distributions," Int. J. Numer. Meth. Fluids, Vol. 56, 2008, pp. 233-255.

${ }^{59}$ Paredes, P., Hermanns, M., Le Clainche, S., and Theofilis, V., "Order $10^{4}$ speedup in global linear instability analysis using matrix formation," Comput. Meth. Appl. Mech. Eng., Vol. 253, 2013, pp. 287-304.

${ }^{60}$ Choudhari, M., Li, F., and Edwards, J., "Stability analysis of roughness array wake in a high-speed boundary layer," AIAA Paper 2009-0170, 2009.

${ }^{61}$ Choudhari, M., Li, F., Chang, C., Edwards, J., Kegerise, M., and King, R., "Laminar-turbulent transition behind dicrete roughness elements in a high-speed boundary layer," AIAA Paper 2013-0081, 2013.

${ }^{62}$ Choudhari, M., Li, F., Chang, C., Norris, A., and Edwards, J., "Wake instabilities behind discrete roughness elements in high speed boundary layers," AIAA Paper 2013-0081, 2013.

${ }^{63}$ Paredes, P., Choudhari, M., and Li, F., "Nonlinear transient growth and boundary layer transition," AIAA Paper 20163956, 2016.

${ }^{64}$ Paredes, P., Choudhari, M., and Li, F., "Transition due to streamwise streaks in a supersonic flat plate boundary layer," Phys. Rev. Fluids, Vol. 1, 2016, pp. 083601-1-23.

${ }^{65}$ Paredes, P., Choudhari, M., and Li, F., "Transient growth and streak instabilities on a hypersonic blunt body," AIAA Paper 2017-0066, 2017.

${ }^{66}$ Jewell, J., Kennedy, R., Laurence, S., and Kimmel, R., "Transition on a variable bluntness 7-degree cone at high Reynolds number," AIAA Paper 2018-0000, 2018. 